\title{
Stereoelectronic Effects in Diastereoselective Formation of Fulleroids
}

\author{
Hiroshi Kitamura, Ken Kokubo and Takumi Oshima* \\ Department of Applied Chemistry, Graduate School of Engineering, Osaka University, \\ Suita, Osaka 565-0871, Japan \\ E-Mail: oshima@chem.eng.osaka-u.ac.jp
}

\section{Contents}

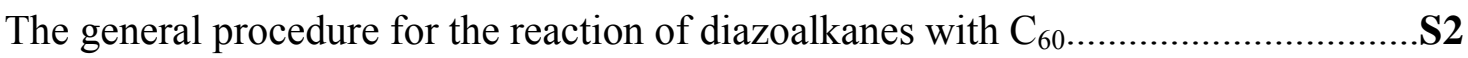

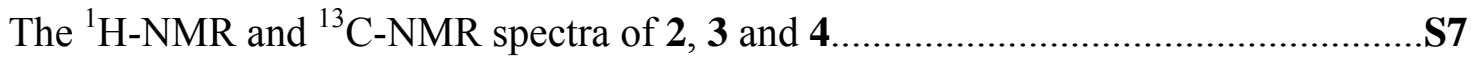

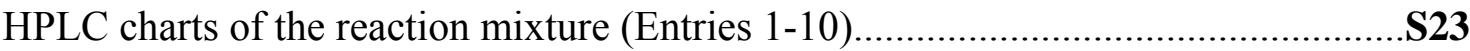

The ${ }^{1} \mathrm{H}-\mathrm{NMR}$ spectra for the determination of product ratios (Entries 1-10)..............S25

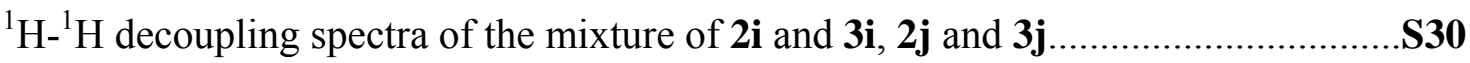




\section{A. General procedure for the reaction of unstable diazoalkanes (1a-c, $1 \mathrm{~g}-\mathrm{h})$ with $\mathrm{C}_{60}$}

To a solution of $\mathrm{C}_{60}(36 \mathrm{mg}, 0.05 \mathrm{mmol})$ and the corresponding hydrazones (1.2-2.8 eq.) in o-dichlorobenzene $(3 \mathrm{~mL})$ was added powdered $\mathrm{Ag}_{2} \mathrm{O}$ (1.1 eq.). The reaction solution was stirred for $2-12 \mathrm{~h}$ at room temperature in the dark. The reaction was monitored by HPLC on Buckyprep column with toluene. The reaction mixture was loaded onto Florisil column and eluted with toluene to remove the inorganic materials. Then, monoadducts $\mathbf{2 ,} 3$ and $\mathbf{4}$ were clearly separated from $\mathrm{C}_{60}$ as well as multiadducts by HPLC on Buckyprep column with toluene. The total yields of the monoadducts 2, 3, and 4 were in the range of $9-36 \%$. The fulleroids $(2+3)$ and methanofullerene 4 were separated by the short-time recycle HPLC treatment $(\sim 1 \mathrm{~h})$ on Buckyprep column with toluene. For the cases of 1a and $\mathbf{1 h}$, the long-time recycle HPLC treatment (1d) gave the enriched isomer ratio (\%); 2a $65 \rightarrow 86 \%$, $2 \mathbf{h} 52 \rightarrow 91 \%$ and $\mathbf{3 h} 48 \rightarrow 99 \%$. Incidentally, no isomerization of $\mathbf{2}, \mathbf{3} \rightarrow$ 4 was observed as confirmed by HPLC as well as ${ }^{1} \mathrm{H}-\mathrm{NMR}$.

\section{B. General procedure for the reaction of stable diazoalkanes $(1 \mathrm{~d}-\mathrm{f}, \mathbf{1} \mathrm{i}-\mathbf{j})$ with $\mathrm{C}_{60}$}

Monotolyl- and diaryl-substituted diazoalkanes $(\mathbf{1} \mathbf{d}-\mathbf{f}, \mathbf{1} \mathbf{i}-\mathbf{j})$ were prepared from the corresponding hydrazones with $\mathrm{HgO}$ according to the literature procedure. ${ }^{1}$ The concentrations of monotolyldiazoalkanes solution were determined by titration of $p$-chlorobenzoic acid. Diaryldiazomethanes were purified by recrystallization from hexane and used as a toluene solution. To a solution of $\mathrm{C}_{60}(36 \mathrm{mg}$, $0.05 \mathrm{mmol})$ in $o$-dichlorobenzene $(3 \mathrm{~mL})$ was added the prepared diazoalkane solution $(0.5-0.75 \mathrm{eq}$.). The reaction solution was stirred for $2-12 \mathrm{~h}$ at room temperature in the dark. The reaction was monitored by HPLC on Buckyprep column with toluene. Monoadducts were separated from $\mathrm{C}_{60}$ as well as multiadducts by HPLC on Buckyprep column with toluene. The total yields of monoadducts were in the range of $23-42 \%$. The fulleroids $(2+3)$ and methanofullerene 4 were separated by the short-time recycle HPLC treatment ( $\sim$ h) on Buckyprep column with toluene. For the cases of 1f, the long-time recycle HPLC treatment (1d) gave the enriched isomer ratio (\%); 3f $62 \rightarrow 88 \%$. Incidentally, no isomerization of $\mathbf{2}, \mathbf{3} \rightarrow \mathbf{4}$ was observed as confirmed by HPLC as well as ${ }^{1} \mathrm{H}-\mathrm{NMR}$.

${ }^{1}$ Oshima, T; Yoshioka, A.; Nagai, T. J. Chem. Soc. Perkin 2, 1978, 1283-1288. 
Compound 2a (enriched isomer ratio 86\%): LC-MS (APPI) $\mathrm{m} / \mathrm{z} 776 ;{ }^{1} \mathrm{H}-\mathrm{NMR}\left(1: 1 \mathrm{CS}_{2}: \mathrm{CDCl}_{3}, 270\right.$ MHz) $\delta 3.69$ (q, $J=7.4 \mathrm{~Hz}, 2 \mathrm{H}), 1.50$ (t, $J=7.4 \mathrm{~Hz}, 3 \mathrm{H}), 1.12$ (s, 3H); ${ }^{13} \mathrm{C}-\mathrm{NMR}\left(1: 1 \mathrm{CS}_{2}: \mathrm{CDCl}_{3}\right.$, $67.5 \mathrm{MHz}) \delta 147.21$ (2C), 144.87 (2C), 144.52 (2C), 144.15 (1C), 143.78 (2C), 143.64 (2C), 143.50 (2C), 143.43 (1C), 143.37 (2C), 143.24 (2C), 142.93 (2C), 142.81 (2C), 142.72 (2C), 142.66 (2C), 142.53 (2C), 142.46 (4C), 142.43 (2C), 142.02 (2C), 141.89 (4C), 141.69 (2C), 141.64 (2C), 141.18 (2C), 140.73 (2C), 139.91 (1C), 139.60 (1C), 139.41 (2C), 138.47 (2C), 138.44 (2C), 137.53 (2C), 136.40 (2C), 134.79 (2C), 49.35 (1C), $35.30\left(\mathrm{CH}_{2}\right), 19.05\left(\mathrm{CH}_{3}\right), 10.09\left(\mathrm{CH}_{3}\right) ; \mathrm{UV} / \mathrm{vis}$ (o-dichlorobenzene) $\lambda_{\max } 543,605 \mathrm{~nm}$ (mixture of 2a:3a $=6: 1$ ).

Compound 3a: ${ }^{1} \mathrm{H}-\mathrm{NMR}\left(1: 1 \mathrm{CS}_{2}: \mathrm{CDCl}_{3}, 270 \mathrm{MHz}\right) \delta 3.09$ (s, 3H), $1.4(\mathrm{~m}, 2 \mathrm{H}), 0.96(\mathrm{t}, J=7.3$ $\mathrm{Hz}, 3 \mathrm{H})$.

Compound 2b (enriched isomer ratio 91\%): LC-MS (APPI) m/z 790; ${ }^{1} \mathrm{H}-\mathrm{NMR}\left(1: 1 \mathrm{CS}_{2}: \mathrm{CDCl}_{3}\right.$, $270 \mathrm{MHz}) \delta 5.13$ (quint, $J=6.7 \mathrm{~Hz}, 1 \mathrm{H}), 1.42(\mathrm{~d}, J=6.7 \mathrm{~Hz}, 6 \mathrm{H}), 0.93(\mathrm{~s}, 3 \mathrm{H}) ;{ }^{13} \mathrm{C}-\mathrm{NMR}(1: 1$ $\left.\mathrm{CS}_{2}: \mathrm{CDCl}_{3}, 67.5 \mathrm{MHz}\right) \delta 147.11(2 \mathrm{C}), 144.84$ (2C), 144.49 (2C), 144.12 (1C), $143.76(2 \mathrm{C}), 143.59$ (2C), 143.41 (2C), 143.22 (2C), 142.91 (4C), 142.84 (2C), 142.81 (2C), 142.76 (2C), 142.68 (2C), 142.54 (2C), 142.32 (2C), 141.98 (3C), 141.77 (2C), 141.62 (2C), 141.54 (2C), 141.06 (2C), 140.53 (2C), 139.87 (2C), 139.60 (2C), 138.56 (2C), 138.47 (2C), 137.43 (2C), 135.95 (3C), 135.16 (1C), $134.82(2 \mathrm{C}), 53.22(1 \mathrm{C}), 36.99(\mathrm{CH}), 18.20\left(2 \mathrm{CH}_{3}\right), 13.26\left(\mathrm{CH}_{3}\right)$; UV/vis (o-dichlorobenzene) $\lambda_{\max }$ $547,606 \mathrm{~nm}$ (mixture of $\mathbf{2 b}: 3 \mathbf{b}=10: 1)$.

Compound 3b: ${ }^{1} \mathrm{H}-\mathrm{NMR}\left(1: 1 \mathrm{CS}_{2}: \mathrm{CDCl}_{3}, 270 \mathrm{MHz}\right) \delta 2.98(\mathrm{~s}, 3 \mathrm{H}), 1.81$ (quint, $J=6.8 \mathrm{~Hz}, 1 \mathrm{H}$ ), $0.93(\mathrm{~d}, J=6.7 \mathrm{~Hz}, 6 \mathrm{H})$.

Compound 2c (enriched isomer 95\%): LC-MS (APPI) $\mathrm{m} / \mathrm{z}$ 804; ${ }^{1} \mathrm{H}-\mathrm{NMR}\left(1: 1 \quad \mathrm{CS}_{2}: \mathrm{CDCl}_{3}, 270\right.$ MHz) $\delta 1.79(\mathrm{~s}, 9 \mathrm{H}), 1.13(\mathrm{~s}, 3 \mathrm{H}) ;{ }^{13} \mathrm{C}-\mathrm{NMR}\left(1: 1 \mathrm{CS}_{2}: \mathrm{CDCl}_{3}, 67.5 \mathrm{MHz}\right) \delta 147.43(2 \mathrm{C}), 145.37$ (2C), 145.08 (2C), 144.51 (2C), 144.02 (1C), 143.68 (2C), 143.58 (2C), 143.35 (3C), 143.30 (2C), 143.27 (2C), 142.85 (2C), 142.74 (2C), 142.52 (2C), 142.31 (2C), 142.15 (4C), 141.68 (2C), 141.61 (2C), 141.52 (2C), 141.41 (2C), 140.90 (2C), 140.47 (2C), 140.32 (2C), 139.66 (1C), 138.03 (3C), 137.93 (2C), 137.29 (2C), 137.01 (2C), 134.31 (2C), 130.18 (2C), 58.35 (1C), 42.01 (q-C), 31.85 $\left(3 \mathrm{CH}_{3}\right), 20.90\left(\mathrm{CH}_{3}\right)$; UV/vis (o-dichlorobenzene) $\lambda_{\max } 550,610 \mathrm{~nm}$ (mixture of $2 \mathrm{c}: 3 \mathrm{c}=19: 1$ ).

Compound 3c: ${ }^{1} \mathrm{H}-\mathrm{NMR}\left(1: 1 \mathrm{CS}_{2}: \mathrm{CDCl}_{3}, 270 \mathrm{MHz}\right) \delta 3.40(\mathrm{~s}, 3 \mathrm{H}), 1.17(\mathrm{~s}, 9 \mathrm{H})$.

Compound 4c: ${ }^{1} \mathrm{H}-\mathrm{NMR}\left(1: 1 \mathrm{CS}_{2}: \mathrm{CDCl}_{3}, 270 \mathrm{MHz}\right) \delta 2.37$ (s, 3H), $1.82(\mathrm{~s}, 9 \mathrm{H})$.

Compound 2d (enriched isomer ratio 89\%): LC-MS (APPI) $\mathrm{m} / \mathrm{z} 838 ;{ }^{1} \mathrm{H}-\mathrm{NMR}\left(1: 1 \mathrm{CS}_{2}: \mathrm{CDCl}_{3}, 270\right.$ MHz) $\delta 7.83(\mathrm{~d}, J=8.2 \mathrm{~Hz}, 2 \mathrm{H}), 7.33(\mathrm{~d}, J=8.2 \mathrm{~Hz}, 2 \mathrm{H}), 2.44$ (s, 3H), $1.32(\mathrm{~s}, 3 \mathrm{H}) ;{ }^{13} \mathrm{C}-\mathrm{NMR}(1: 1$ $\left.\mathrm{CS}_{2}: \mathrm{CDCl}_{3}, 67.5 \mathrm{MHz}\right) \delta 147.15$ (2C), 146.50 (2C), 144.84 (2C), $144.46(2 \mathrm{C}), 144.15$ (1C), 143.90 (2C), 143.64 (2C), 143.47 (2C), 143.35 (2C), 143.27 (2C), 142.85 (3C), 142.75 (2C), 142.47 (2C), 142.25 (2C), 141.91 (4C), 141.81 (2C), 141.71 (2C), 141.29 (3C), 141.14 (2C), 140.82 (2C), 140.16 (2C), 139.36 (2C), 138.51 (2C), 138.02 (3C), 137.93 (2C), 137.68 (2C), 136.84 (3C), 136.40 (1C), $134.82(2 \mathrm{C}), 129.55(2 \operatorname{arom} \mathrm{CH}), 129.03(2 \operatorname{arom~} \mathrm{CH}), 56.41(1 \mathrm{C}), 25.58\left(\mathrm{CH}_{3}\right), 21.36\left(\mathrm{CH}_{3}\right)$; 
$\mathrm{UV} /$ vis (o-dichlorobenzene) $\lambda_{\max } 545,602 \mathrm{~nm}$ (mixture of 2d:3d $=8: 1$ ).

Compound 3d: ${ }^{1} \mathrm{H}-\mathrm{NMR}\left(1: 1 \mathrm{CS}_{2}: \mathrm{CDCl}_{3}, 270 \mathrm{MHz}\right) \delta$ 7.17-7.00(m, 4H), 3.19 (s, 3H), 2.31 (s, $3 \mathrm{H})$.

Compound 4d: LC-MS (APPI) m/z 838; ${ }^{1} \mathrm{H}-\mathrm{NMR}\left(1: 1 \mathrm{CS}_{2}: \mathrm{CDCl}_{3}, 270 \mathrm{MHz}\right) \delta 7.79$ (d, J=8.1 Hz, 2H), $7.29(\mathrm{~d}, J=8.1 \mathrm{~Hz}, 2 \mathrm{H}), 2.51(\mathrm{~s}, 3 \mathrm{H}), 2.45(\mathrm{~s}, 3 \mathrm{H}) ;{ }^{13} \mathrm{C}-\mathrm{NMR}\left(1: 1 \mathrm{CS}_{2}: \mathrm{CDCl}_{3}, 67.5 \mathrm{MHz}\right) \delta$ 148.51 (2C), 147.75 (2C), 145.54 (2C), 144.83 (5C), 144.75 (6C), 144.68 (2C), 144.43 (4C), 144.32 (2C), 144.09 (2C), 143.68 (2C), 143.41 (4C), 142.76 (2C), 142.66 (4C), 141.95 (2C), 141.83 (6C), 140.68 (2C), 140.45 (2C), 137.68 (2C), 137.37 (5C), 135.99 (2C), 130.55 (2 arom CH), 129.15 (2 arom $\mathrm{CH}), 80.75(2 \mathrm{C}), 47.23(1 \mathrm{C}), 21.53\left(\mathrm{CH}_{3}\right), 21.42\left(\mathrm{CH}_{3}\right)$; UV/vis (o-dichlorobenzene) $\lambda_{\max } 435$, 493, $699 \mathrm{~nm}$.

Compound 2e: LC-MS (APPI) m/z 866; ${ }^{1} \mathrm{H}-\mathrm{NMR}\left(1: 1 \mathrm{CS}_{2}: \mathrm{CDCl}_{3}, 270 \mathrm{MHz}\right) \delta 7.74$ (d, J=8.1 Hz, 2H), $7.29(\mathrm{~d}, J=8.1 \mathrm{~Hz}, 2 \mathrm{H}), 2.45$ (s, 3H), 1.95 (quint, $J=6.8 \mathrm{~Hz}, 1 \mathrm{H}$ ), 1.44 (d, $J=6.8 \mathrm{~Hz}, 6 \mathrm{H}$ ); ${ }^{13} \mathrm{C}-\mathrm{NMR}\left(1: 1 \mathrm{CS}_{2}: \mathrm{CDCl}_{3}, 67.5 \mathrm{MHz}\right) \delta 146.94$ (2C), 144.90 (2C), 144.39 (2C), 144.15 (1C), 143.92 (2C), 143.63 (2C), 143.38 (2C), 143.25 (2C), 142.74 (2C), 142.71 (2C), 142.57 (2C), 142.50 (2C), 142.20 (2C), 142.15 (2C), 141.84 (2C), 141.55 (2C), 141.19 (2C), 140.88 (3C), 140.79 (2C), 140.30 (2C), 140.14 (2C), 139.69 (4C), 139.41 (3C), 139.13 (2C), 138.67 (3C), 137.46 (2C), 136.04 (1C), $136.92(3 \mathrm{C}), 134.77(2 \mathrm{C}), 132.41(2 \operatorname{arom} \mathrm{CH}), 128.20(2 \operatorname{arom~} \mathrm{CH}), 65.83(1 \mathrm{C}), 29.73(\mathrm{CH})$, $21.37\left(\mathrm{CH}_{3}\right), 18.03\left(2 \mathrm{CH}_{3}\right)$; UV/vis (o-dichlorobenzene) $\lambda_{\max } 429,549,606 \mathrm{~nm}$.

Compound 4e: LC-MS (APPI) m/z 866; ${ }^{1} \mathrm{H}-\mathrm{NMR}\left(1: 1 \mathrm{CS}_{2}: \mathrm{CDCl}_{3}, 270 \mathrm{MHz}\right) \delta 7.67$ (d, J=8.1 Hz, 2H), $7.25(\mathrm{~d}, J=8.1 \mathrm{~Hz}, 2 \mathrm{H}$ ), 3.39 (quint, $J=6.8 \mathrm{~Hz}, 1 \mathrm{H}$ ), 2.46 (s, 3H), $1.40(\mathrm{~d}, J=6.8 \mathrm{~Hz}, 6 \mathrm{H}$ ); ${ }^{13} \mathrm{C}-\mathrm{NMR}\left(1: 1 \mathrm{CS}_{2}: \mathrm{CDCl}_{3}, 67.5 \mathrm{MHz}\right) \delta 148.90$ (2C), 147.61 (2C), 145.72 (4C), 144.87 (2C), 144.84 (2C), 144.78 (2C), 144.74 (2C), 144.52 (1C), 144.50 (2C), 144.35 (2C), 144.17 (2C), 144.14 (2C), 143.72 (2C), 143.50 (3C), 142.85 (1C), 142.72 (4C), 142.63 (4C), 141.91 (2C), 141.86 (4C), 141.83 (2C), 140.69 (1C), 140.40 (2C), 140.30 (1C), 138.05 (2C), 137.57 (3C), 137.37 (2C), 133.57 (2 arom $\mathrm{CH}), 129.60(2 \mathrm{C}), 128.12(2 \operatorname{arom} \mathrm{CH}), 80.68(2 \mathrm{C}), 57.59(1 \mathrm{C}), 29.72(\mathrm{CH}), 21.46\left(\mathrm{CH}_{3}\right)$, $19.67\left(2 \mathrm{CH}_{3}\right) ; \mathrm{UV} /$ vis (o-dichlorobenzene) $\lambda_{\max } 434,494,700 \mathrm{~nm}$.

Compound 2f: ${ }^{1} \mathrm{H}-\mathrm{NMR}\left(1: 1 \mathrm{CS}_{2}: \mathrm{CDCl}_{3}, 270 \mathrm{MHz}\right) \delta 7.69(\mathrm{~d}, J=8.1 \mathrm{~Hz}, 2 \mathrm{H}), 7.29(\mathrm{~d}, J=8.1 \mathrm{~Hz}$, $2 \mathrm{H}), 2.43(\mathrm{~s}, 3 \mathrm{H}), 1.03(\mathrm{tt}, J=8.2,5.3 \mathrm{~Hz}, 1 \mathrm{H}), 0.33-0.29(\mathrm{~m}, 2 \mathrm{H}), 0.05-0.00(\mathrm{~m}, 2 \mathrm{H})$.

Compound 3f: (enriched isomer ratio 88\%) LC-MS (APPI) $\mathrm{m} / \mathrm{z} 864 ;{ }^{1} \mathrm{H}-\mathrm{NMR}\left(1: 1 \mathrm{CS}_{2}: \mathrm{CDCl}_{3}, 270\right.$ MHz) $\delta 6.99(\mathrm{~m}, 4 \mathrm{H}), 3.93(\mathrm{tt}, J=8.2,5.3 \mathrm{~Hz}, 1 \mathrm{H}), 2.30$ (s, 3H), 0.88-0.82 (m, 2H), 0.33-0.27 (m, $2 \mathrm{H}) ;{ }^{13} \mathrm{C}-\mathrm{NMR}\left(1: 1 \mathrm{CS}_{2}: \mathrm{CDCl}_{3}, 67.5 \mathrm{MHz}\right) \delta 147.10$ (2C), $144.80(2 \mathrm{C}), 144.37$ (1C), 143.71 (2C), 143.47 (2C), 143.34 (2C), 143.17 (2C), 143.12 (2C), 142.80 (2C), 142.71 (3C), 142.52 (2C), 142.24 (2C), 142.11 (2C), 141.77 (2C), 141.68 (2C), 141.53 (2C), 141.02 (2C), 140.46 (1C), 139.83 (4C), 138.42 (3C), 137.51 (2C), 136.89 (2C), 136.55 (2C), 136.00 (1C), 134.06 (1C), 133.95 (2C), 128.49 (2 arom $\mathrm{CH}), 127.81(2$ arom $\mathrm{CH}), 57.55(1 \mathrm{C}), 24.17(\mathrm{CH}), 21.23\left(\mathrm{CH}_{3}\right), 4.07\left(2 \mathrm{CH}_{2}\right)$; UV/vis (o-dichlorobenzene) $\lambda_{\max } 547,608 \mathrm{~nm}$ (mixture of $\mathbf{2 f : 3 f}=1: 7.3$ ). 
Compound 4f: LC-MS (APPI) m/z 864; ${ }^{1} \mathrm{H}-\mathrm{NMR}\left(1: 1 \mathrm{CS}_{2}: \mathrm{CDCl}_{3}, 270 \mathrm{MHz}\right) \delta 7.64$ (d, J=8.1 Hz, 2H), 7.25 (d, $J=8.1 \mathrm{~Hz}, 2 \mathrm{H}), 2.48(\mathrm{~m}, 1 \mathrm{H}), 2.44(\mathrm{~s}, 3 \mathrm{H}), 0.87-0.82(\mathrm{~m}, 2 \mathrm{H}), 0.63-0.57(\mathrm{~m}, 2 \mathrm{H})$; ${ }^{13} \mathrm{C}-\mathrm{NMR}\left(1: 1 \mathrm{CS}_{2}: \mathrm{CDCl}_{3}, 67.5 \mathrm{MHz}\right) \delta 148.80$ (2C), 148.14 (4C), 145.68 (2C), 144.85 (3C), 144.82 (4C), 144.76 (4C), 144.66 (2C), 144.40 (2C), 144.29 (2C), 144.14 (3C), 144.07 (2C), 143.68 (2C), 143.50 (2C), 143.45 (2C), 142.71 (3C), 142.64 (4C), 141.88 (2C), 141.79 (4C), 140.66 (1C), 140.46 (2C), 137.80 (2C), 137.74(3C), 137.25 (1C), 133.04 (2 arom CH), 129.87 (2C), 128.46 (2 arom $\mathrm{CH}), \quad 80.95(2 \mathrm{C}), \quad 52.36(1 \mathrm{C}), \quad 21.45 \quad\left(\mathrm{CH}_{3}\right), \quad 13.94 \quad(\mathrm{CH}), \quad 3.71 \quad\left(2 \mathrm{CH}_{2}\right) ; \quad \mathrm{UV} /$ vis (o-dichlorobenzene) $\lambda_{\max } 434,494,700 \mathrm{~nm}$.

Compound 2g: LC-MS (APPI) m/z 774; ${ }^{1} \mathrm{H}-\mathrm{NMR}\left(1: 1 \mathrm{CS}_{2}: \mathrm{CDCl}_{3}, 270 \mathrm{MHz}\right) \delta 3.20$ (dtt, $J=9.6$, 8.1, $4.8 \mathrm{~Hz}, 1 \mathrm{H}), 1.93(\mathrm{~d}, J=9.6 \mathrm{~Hz}, 2 \mathrm{H}), 1.08-1.01(\mathrm{~m}, 2 \mathrm{H}), 0.64-0.58(\mathrm{~m}, 2 \mathrm{H}) ;{ }^{13} \mathrm{C}-\mathrm{NMR}(1: 1$ $\left.\mathrm{CS}_{2}: \mathrm{CDCl}_{3}, 67.5 \mathrm{MHz}\right) ;{ }^{13} \mathrm{C}-\mathrm{NMR}\left(1: 1 \mathrm{CS}_{2}: \mathrm{CDCl}_{3}, 270 \mathrm{MHz}\right) \delta 147.56(2 \mathrm{C}), 144.56(2 \mathrm{C}), 144.48$ (1C), 143.89 (1C), 143.58 (2C), 143.33 (4C), 143.30 (2C), 143.19 (2C), 143.00 (2C), 142.95 (2C), 142.82 (4C), 142.80 (4C), 142.75 (2C), 142.73 (2C), 142.40 (2C), 142.11 (2C), 141.78 (4C), 141.22 (2C), 139.90 (4C), 139.65 (1C), 138.44 (2C), 138.19 (2C), 137.80 (2C), 137.60 (2C), 137.48 (1C), $134.75(2 \mathrm{C}), 133.69(2 \mathrm{C}), 53.58(\mathrm{CH}), 15.33(\mathrm{CH}), 5.52\left(2 \mathrm{CH}_{2}\right)$; UV/vis (o-dichlorobenzene) $\lambda_{\max }$ $543,605 \mathrm{~nm}$.

Compound 2h (enriched isomer ratio 91\%): LC-MS (APPI) $\mathrm{m} / \mathrm{z} 788 ;{ }^{1} \mathrm{H}-\mathrm{NMR}\left(1: 1 \mathrm{CS}_{2}: \mathrm{CDCl}_{3}, 270\right.$ MHz): $\delta 3.55(\mathrm{tt}, J=8.4,5.8 \mathrm{~Hz}, 1 \mathrm{H}), 1.12-1.03(\mathrm{~m}, 2 \mathrm{H}), 1.00-0.93(\mathrm{~m}, 2 \mathrm{H}), 0.84(\mathrm{~s}, 3 \mathrm{H})$; ${ }^{13} \mathrm{C}-\mathrm{NMR}\left(1: 1 \mathrm{CS}_{2}: \mathrm{CDCl}_{3}, 67.5 \mathrm{MHz}\right) \delta 147.16$ (2C), 144.81 (2C), 144.43 (2C), 144.07 (2C), 143.69 (2C), 143.50 (2C), 143.45 (2C), 143.36 (1C), 143.22 (2C), 143.14 (2C), 142.81 (4C), 142.76 (2C), 142.68 (2C), 142.57 (2C), 142.37 (2C), 141.92 (2C), 141.84 (2C), 141.65 (2C), 141.59 (2C), 141.10 (2C), 140.74 (2C), 139.89 (1C), 139.82 (2C), 139.33 (2C), 138.34 (2C), 138.19 (2C), 137.47 (2C), $137.31(1 \mathrm{C}), 137.13(3 \mathrm{C}), 134.74(2 \mathrm{C}), 48.06(1 \mathrm{C}), 24.29\left(\mathrm{CH}_{3}\right), 16.17(\mathrm{CH}), 4.99\left(2 \mathrm{CH}_{2}\right)$; $\mathrm{UV} / \mathrm{vis}$ (o-dichlorobenzene) $\lambda_{\max } 542,602 \mathrm{~nm}$.

Compound 3h (enriched isomer ratio 99\%): LC-MS (APPI) $\mathrm{m} / \mathrm{z} 788 ;{ }^{1} \mathrm{H}-\mathrm{NMR}\left(1: 1 \mathrm{CS}_{2}: \mathrm{CDCl}_{3}, 270\right.$ MHz) $\delta 2.74(\mathrm{~s}, 3 \mathrm{H}), 0.92(\mathrm{tt}, J=8.1,5.9 \mathrm{~Hz}, 1 \mathrm{H}), 0.62-0.46(\mathrm{~m}, 4 \mathrm{H}),{ }^{13} \mathrm{C}-\mathrm{NMR}\left(1: 1 \mathrm{CS}_{2}: \mathrm{CDCl}_{3}\right.$, $67.5 \mathrm{MHz}) \delta 147.19$ (2C), 144.81 (2C), 144.71 (1C), 144.47 (2C), 143.70 (2C), 143.56 (2C), 143.45 (1C), 143.37 (2C), 143.34 (2C), 143.14 (2C), 142.97 (4C), 142.87 (2C), 142.75 (2C), 142.69 (1C), 142.63 (2C), 142.57 (2C), 142.33 (2C), 141.87 (2C), 141.80 (1C), 141.59 (4C), 141.57 (2C), 141.08 (2C), 140.65 (2C), 139.83 (4C), 139.80 (2C), 138.42 (2C), 138.36 (2C), 137.43 (2C), 136.91 (2C), $134.70(2 \mathrm{C}), 47.30(1 \mathrm{C}), 22.39\left(\mathrm{CH}_{3}\right), 15.84(\mathrm{CH}), 2.92\left(2 \mathrm{CH}_{2}\right)$; UV/vis (o-dichlorobenzene) $\lambda_{\max }$ $544,602 \mathrm{~nm}$.

Compound 4h: LC-MS (APPI) $\mathrm{m} / \mathrm{z}$ 788; ${ }^{1} \mathrm{H}-\mathrm{NMR}\left(1: 1 \mathrm{CS}_{2}: \mathrm{CDCl}_{3}, 270 \mathrm{MHz}\right) \delta 2.26$ (tt, $J=8.4$, $5.8 \mathrm{~Hz}, 1 \mathrm{H}), 1.94(\mathrm{~s}, 3 \mathrm{H}), 1.10-1.04(\mathrm{~m}, 2 \mathrm{H}), 0.98-0.90(\mathrm{~m}, 2 \mathrm{H})$; UV/vis (o-dichlorobenzene) $\lambda_{\max }$ 436, 493, $697 \mathrm{~nm}$.

Compound 2i: ${ }^{1} \mathrm{H}-\mathrm{NMR}\left(1: 1 \mathrm{CS}_{2}: \mathrm{CDCl}_{3}, 270 \mathrm{MHz}\right) \delta 7.90(\mathrm{~d}, J=8.2 \mathrm{~Hz}, 2 \mathrm{H}), 7.3-7.0(\mathrm{~m}, 7 \mathrm{H})$, 
$2.35(\mathrm{~s}, 3 \mathrm{H})$.

Compound 3i: LC-MS (APPI) $\mathrm{m} / \mathrm{z}$ 900; ${ }^{1} \mathrm{H}-\mathrm{NMR}\left(1: 1 \mathrm{CS}_{2}: \mathrm{CDCl}_{3}, 270 \mathrm{MHz}\right) \delta 8.01$ (d, $J=7.6 \mathrm{~Hz}$, 2H), $7.42(\mathrm{t}, J=7.6 \mathrm{~Hz}, 2 \mathrm{H}), 7.3-7.0(\mathrm{~m}, 3 \mathrm{H}), 6.96(\mathrm{~d}, J=7.9 \mathrm{~Hz}, 2 \mathrm{H}), 2.23(\mathrm{~s}, 3 \mathrm{H}) \mathrm{UV} / \mathrm{vis}$ (o-dichlorobenzene) $\lambda_{\max } 543,609 \mathrm{~nm}$ (mixture of $2 \mathbf{i}: 3 \mathbf{i}=1.8: 3$ ).

Compound 4i: LC-MS (APPI) $\mathrm{m} / \mathrm{z}$ 900; ${ }^{1} \mathrm{H}-\mathrm{NMR}\left(1: 1 \mathrm{CS}_{2}: \mathrm{CDCl}_{3}, 270 \mathrm{MHz}\right) \delta 8.01$ (d, J=7.9 Hz, 2H), 7.89 (d, $J=8.2 \mathrm{~Hz}, 2 \mathrm{H}), 7.41(\mathrm{t}, J=7.9 \mathrm{~Hz}, 2 \mathrm{H}), 7.31(\mathrm{t}, J=7.9 \mathrm{~Hz}, 1 \mathrm{H}), 7.21$ (d, $J=8.2 \mathrm{~Hz}$, 2H), 2.39 (s, 3H); ${ }^{13} \mathrm{C}-\mathrm{NMR}\left(1: 1 \mathrm{CS}_{2}: \mathrm{CDCl}_{3}, 67.5 \mathrm{MHz}\right) \delta 147.90$ (4C), 145.03 (4C), 144.84 (4C), 144.77 (4C), 144.37 (4C), 144.28 (2C), 143.96 (4C), 143.50 (4C), 142.67 (2C), 142.61 (8C), 141.88 (4C), 141.80 (4C), 140.57 (4C), 138.85 (2C), 137.96 (2C), 137.88 (1C), 137.56 (2C), 135.70 (2C), $130.56(2$ arom $\mathrm{CH}), 130.54$ (2 arom $\mathrm{CH}), 129.20$ (2 arom $\mathrm{CH}), 128.47$ (arom 2CH), 127.79 (arom $\mathrm{CH}), 78.79$ (2C), 57.89 (1C), $21.38\left(\mathrm{CH}_{3}\right)$; UV/vis (o-dichlorobenzene) $\lambda_{\max } 435,493,700 \mathrm{~nm}$.

Compound 2j: ${ }^{1} \mathrm{H}-\mathrm{NMR}\left(1: 1 \mathrm{CS}_{2}: \mathrm{CDCl}_{3}, 270 \mathrm{MHz}\right) \delta 7.94(\mathrm{~d}, J=8.7 \mathrm{~Hz}, 2 \mathrm{H}), 7.33(\mathrm{~d}, J=7.1 \mathrm{~Hz}$, 2H), 7.17 (t, $J=7.1 \mathrm{~Hz}, 2 \mathrm{H}), 7.09$ (t, $J=7.1 \mathrm{~Hz}, 1 \mathrm{H}), 6.95$ (d, $J=8.7 \mathrm{~Hz}, 2 \mathrm{H}), 3.79$ (s, 3H).

Compound 3j: LC-MS (APPI) m/z 916; ${ }^{1} \mathrm{H}-\mathrm{NMR}\left(1: 1 \mathrm{CS}_{2}: \mathrm{CDCl}_{3}, 270 \mathrm{MHz}\right) \delta 8.01(\mathrm{~d}, J=7.6 \mathrm{~Hz}$, 2H), $7.43(\mathrm{~d}, J=7.6 \mathrm{~Hz}, 2 \mathrm{H}), 7.3-7.25(\mathrm{~m}, 1 \mathrm{H}), 7.26(\mathrm{~d}, J=8.9 \mathrm{~Hz}, 2 \mathrm{H}), 6.68(\mathrm{~d}, J=8.9 \mathrm{~Hz}, 2 \mathrm{H})$, 3.70 (s, 3H); UV/vis (o-dichlorobenzene) $\lambda_{\max } 547,606 \mathrm{~nm}$ (mixture of $\mathbf{2 \mathbf { j }}: \mathbf{3} \mathbf{j}=1: 3$ ).

Compound 4j: LC-MS (APPI) m/z 916; ${ }^{1} \mathrm{H}-\mathrm{NMR}\left(1: 1 \mathrm{CS}_{2}: \mathrm{CDCl}_{3}, 270 \mathrm{MHz}\right) \delta 8.04$ (d, J=8.1 Hz, 2H), 7.96 (d, $J=7.9 \mathrm{~Hz}, 2 \mathrm{H}), 7.44$ (t, $J=7.9 \mathrm{~Hz}, 2 \mathrm{H}), 7.34$ (t, $J=7.8 \mathrm{~Hz}, 1 \mathrm{H}), 6.94$ (d, $J=8.1 \mathrm{~Hz}$, 2H), $3.82(\mathrm{~s}, 3 \mathrm{H}) ;{ }^{13} \mathrm{C}-\mathrm{NMR}\left(1: 1 \mathrm{CS}_{2}: \mathrm{CDCl}_{3}, 67.5 \mathrm{MHz}\right) \delta 158.97$ (1C), 148.03 (2C), 147.11 (4C), 145.08 (8C), 144.93 (4C), 144.83 (4C), 144.44 (4C), 144.35 (4C), 144.04 (4C), 143.58 (4C), 142.74 (2C), 142.68 (8C), 141.99 (2C), 141.97 (4C), 141.87 (2C), 140.62 (2C), 139.09 (2C), 138.04 (2C), $137.93(2 \mathrm{C}), 131.79$ (2 arom $\mathrm{CH}), 130.80(1 \mathrm{C}), 130.55$ (2 arom $\mathrm{CH}), 128.53$ (2 arom $\mathrm{CH}), 128.09$ (1C), 127.79 (arom CH), $113.99(2$ arom $\mathrm{CH}), 79.04(2 \mathrm{C}), 57.60(1 \mathrm{C}), 55.07\left(\mathrm{OCH}_{3}\right)$; UV/vis (o-dichlorobenzene) $\lambda_{\max } 435,496,700 \mathrm{~nm}$. 
${ }^{1} \mathrm{H}-\mathrm{NMR}$ chart $; 2 \mathrm{a}: 3 \mathrm{a}=6: 1$

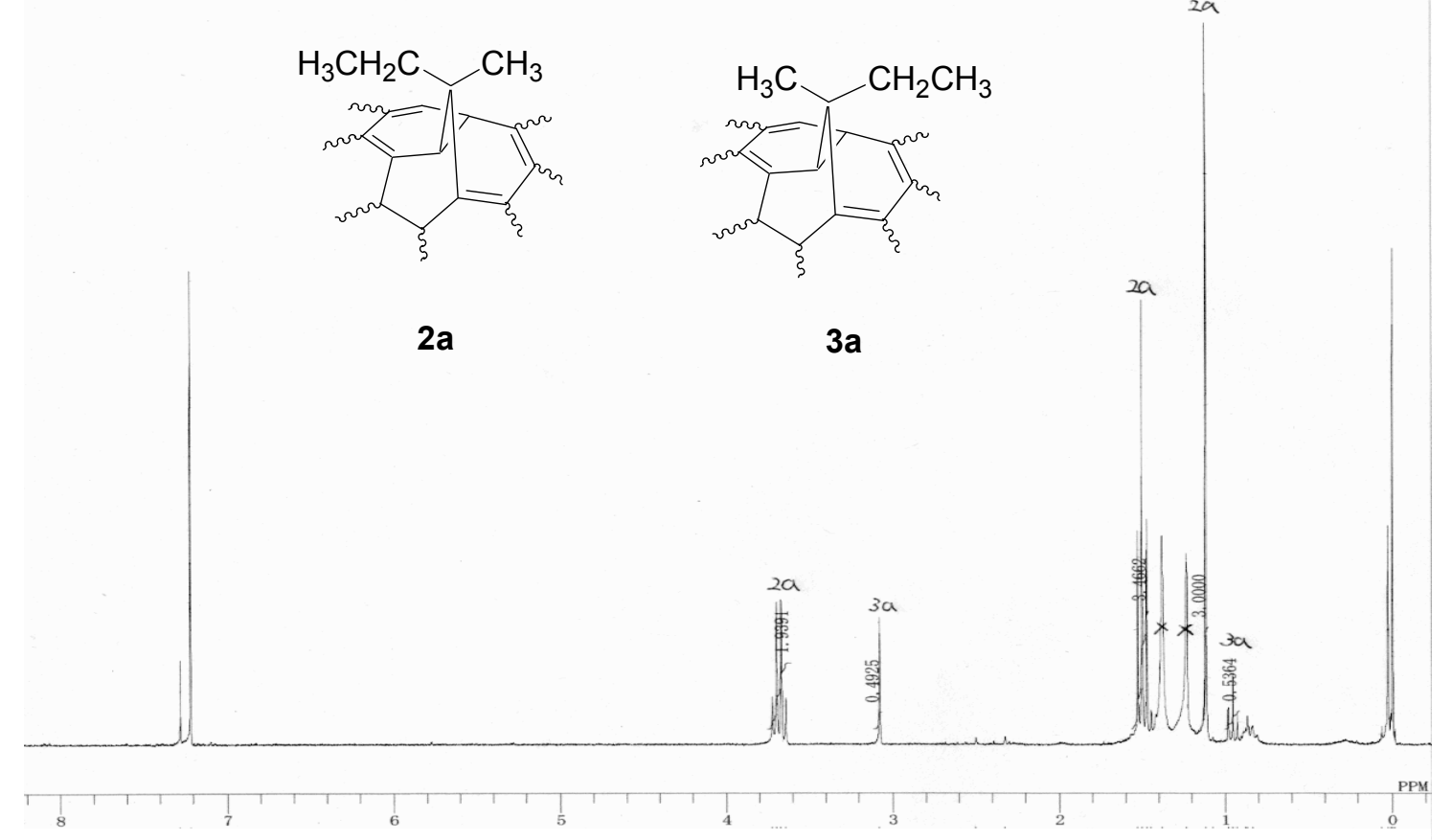

$2 b: 3 b=10: 1$

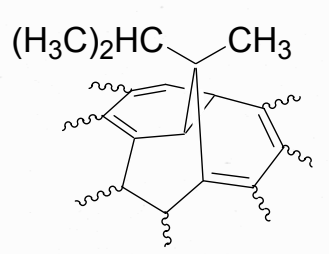

$2 b$

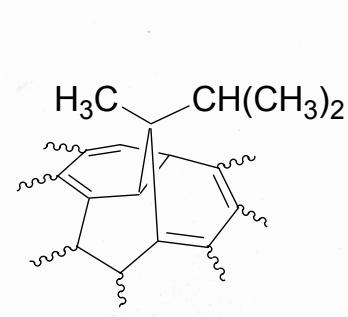

$3 b$

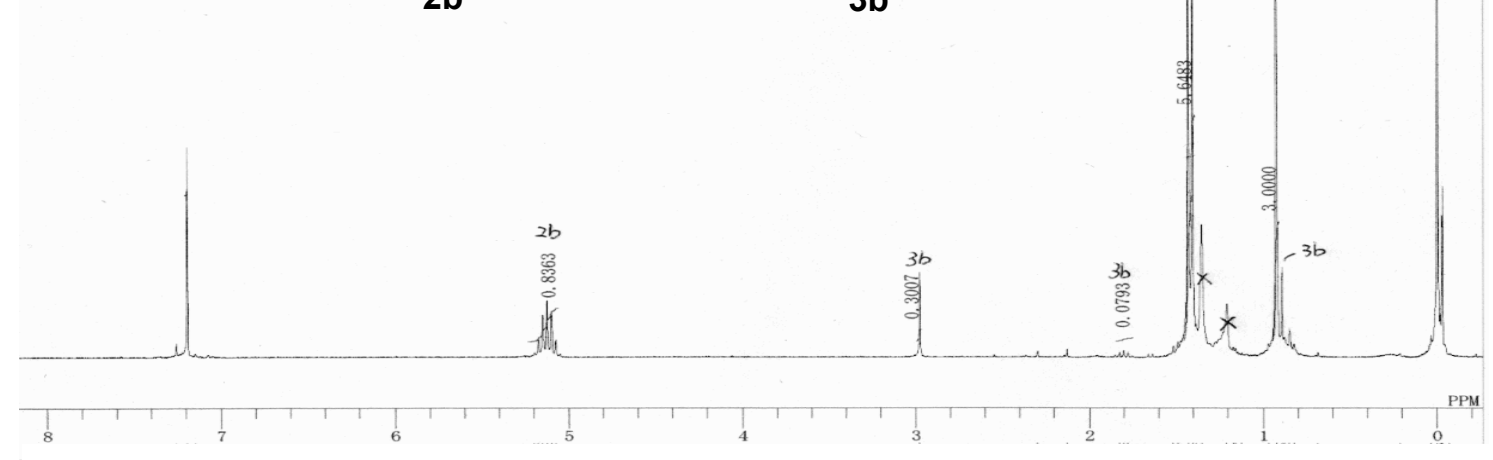




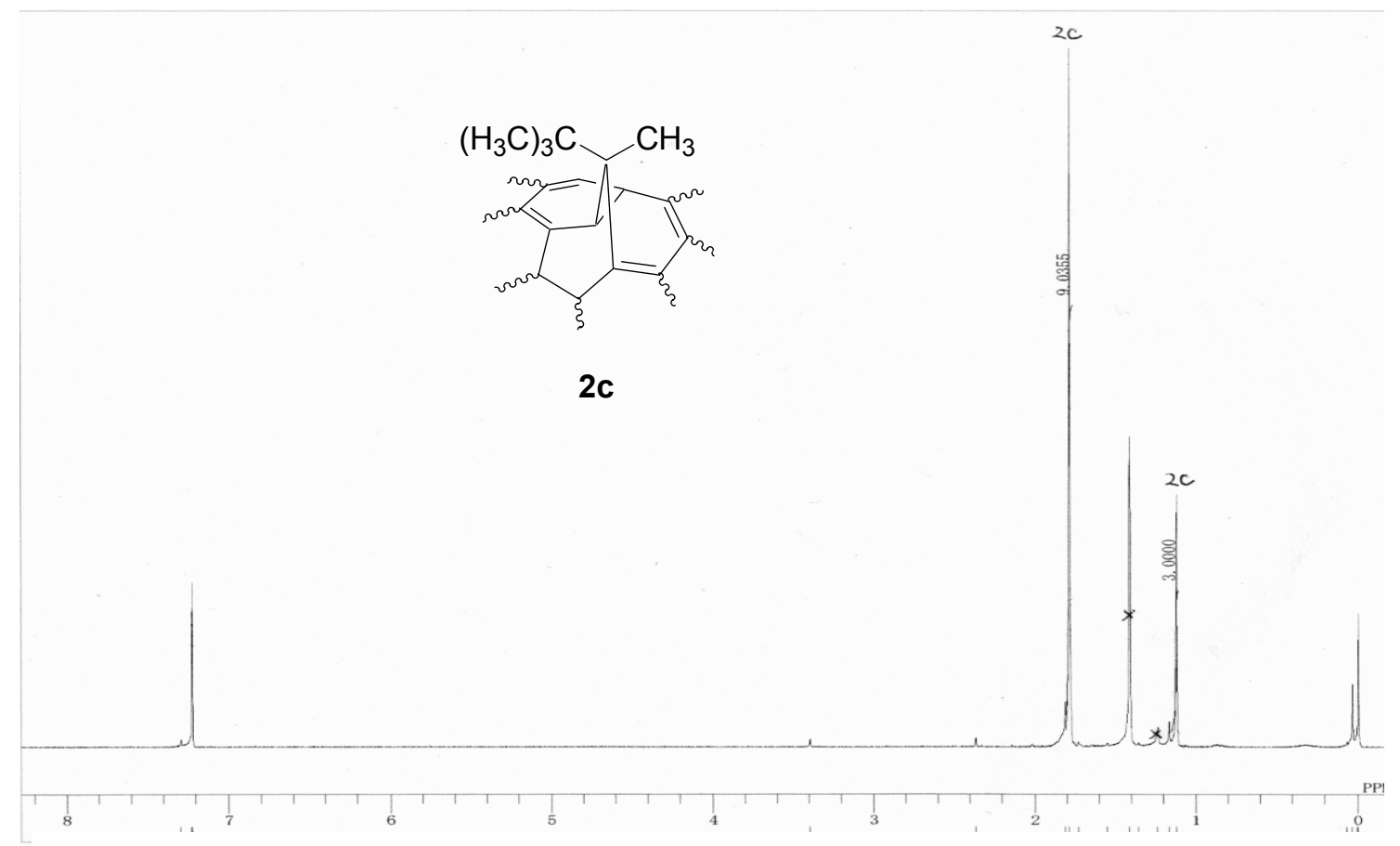

$2 \mathrm{~d}: 3 \mathrm{~d}=8: 1$

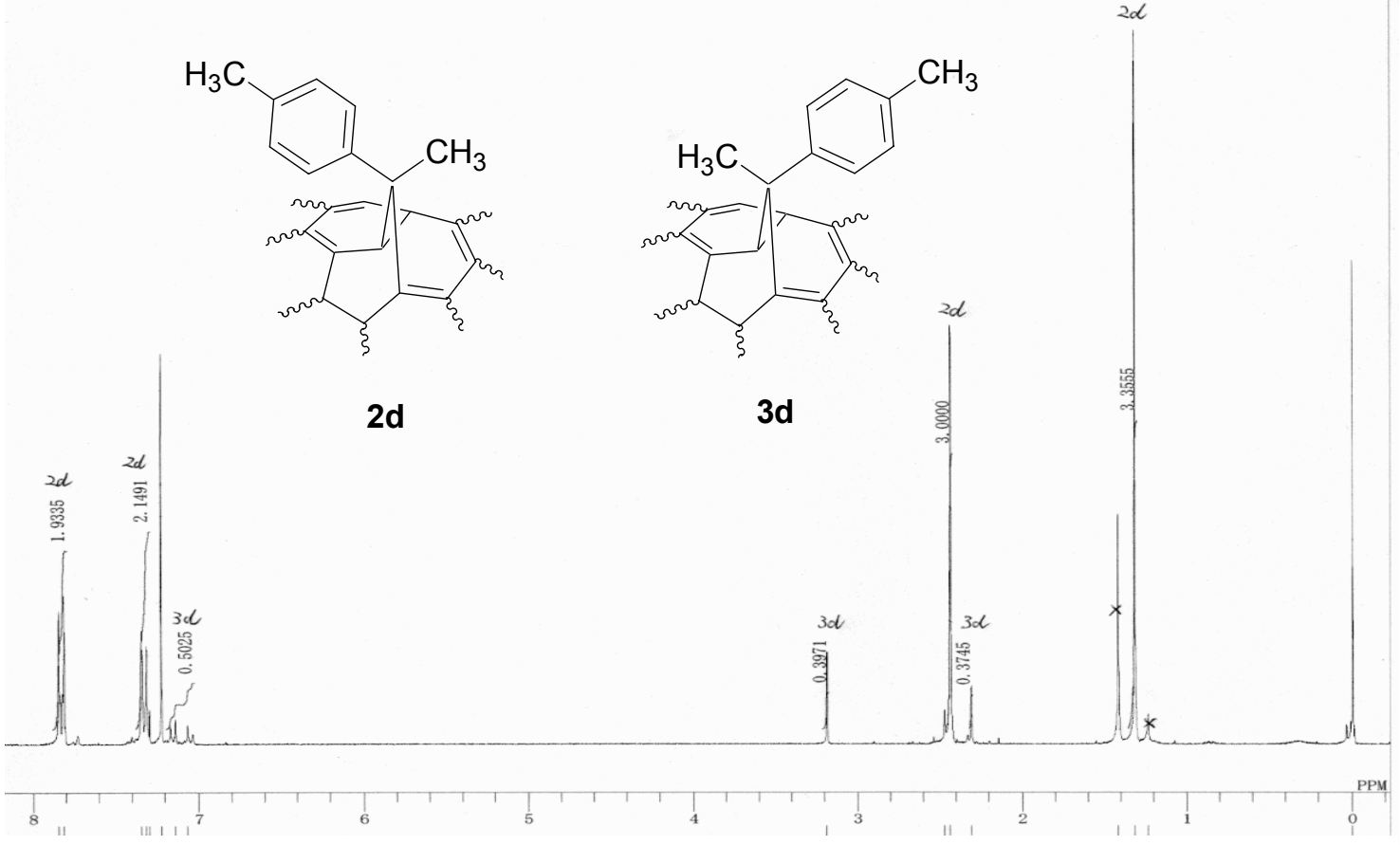



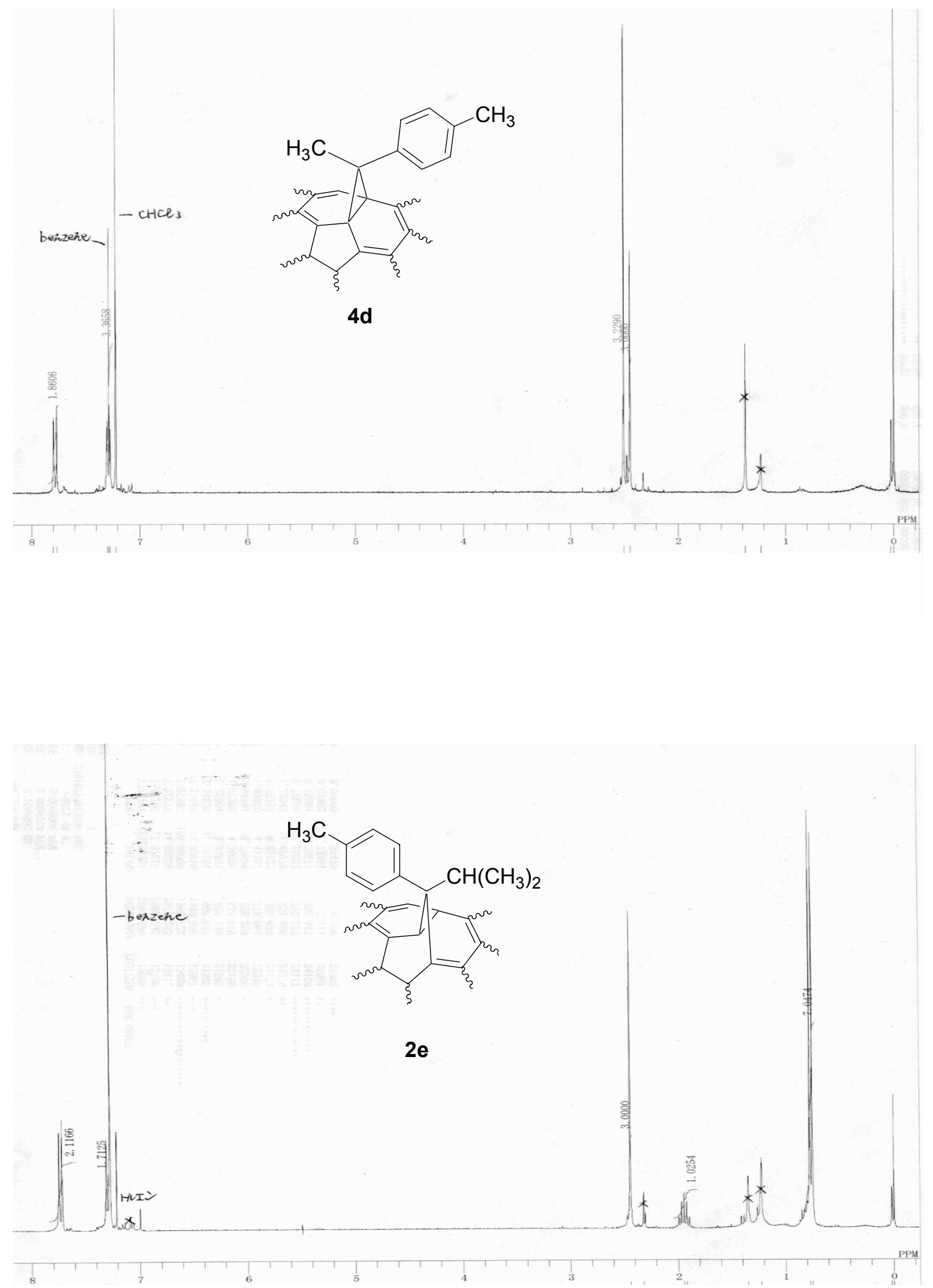


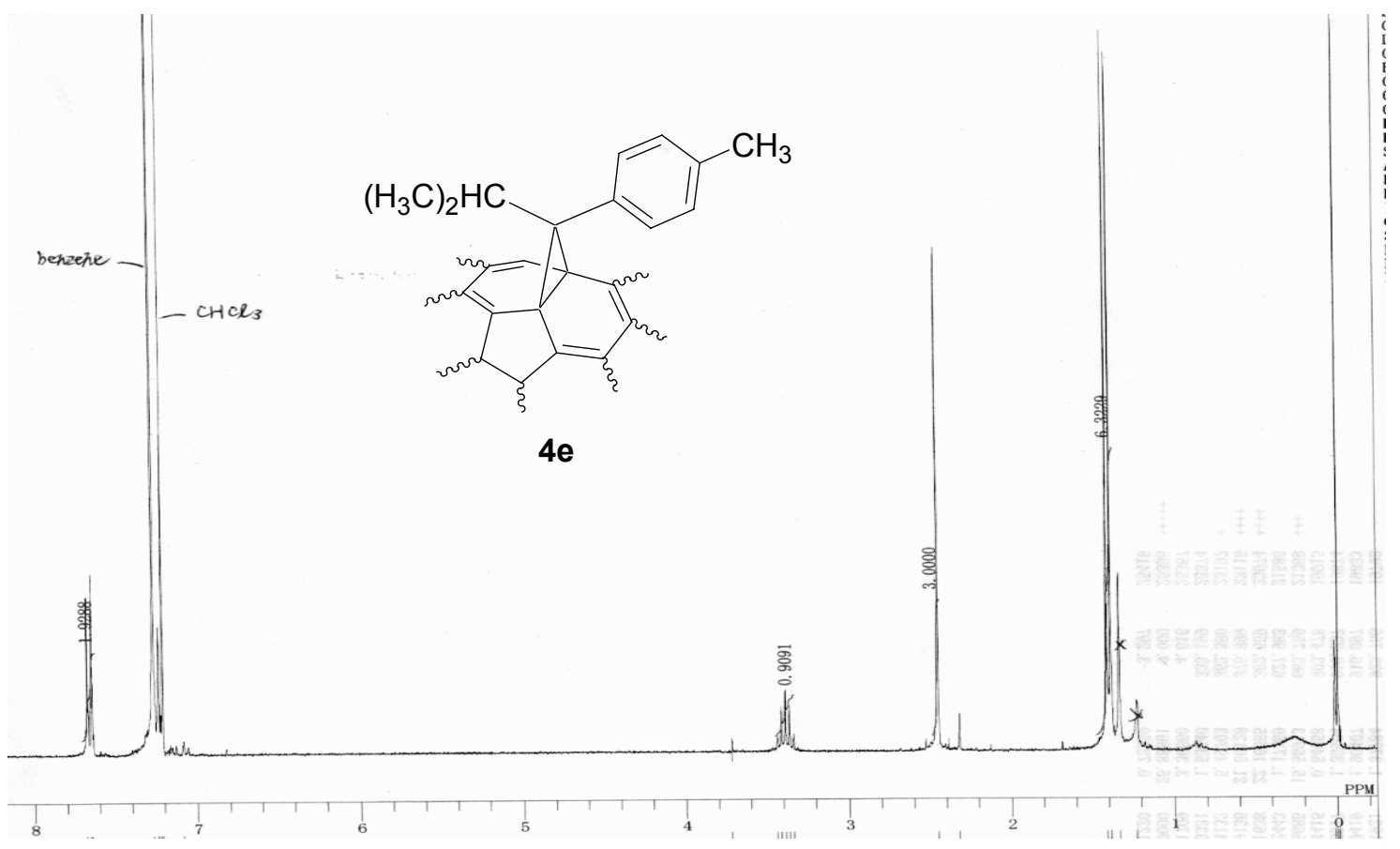

$2 \mathrm{f}: 3 \mathrm{f}=1: 7.3$

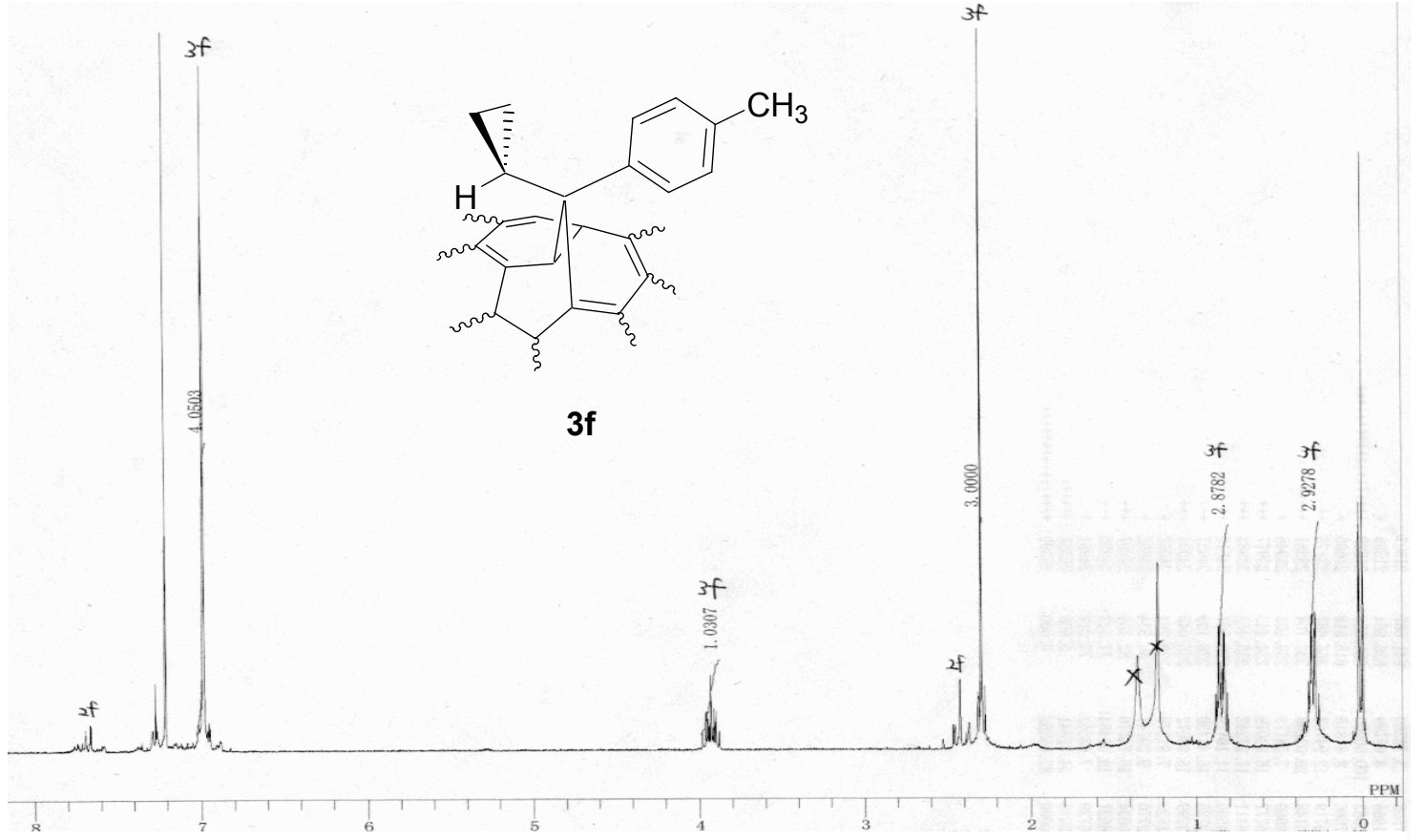


$2 \mathrm{f}: 3 \mathrm{f}=7: 3$
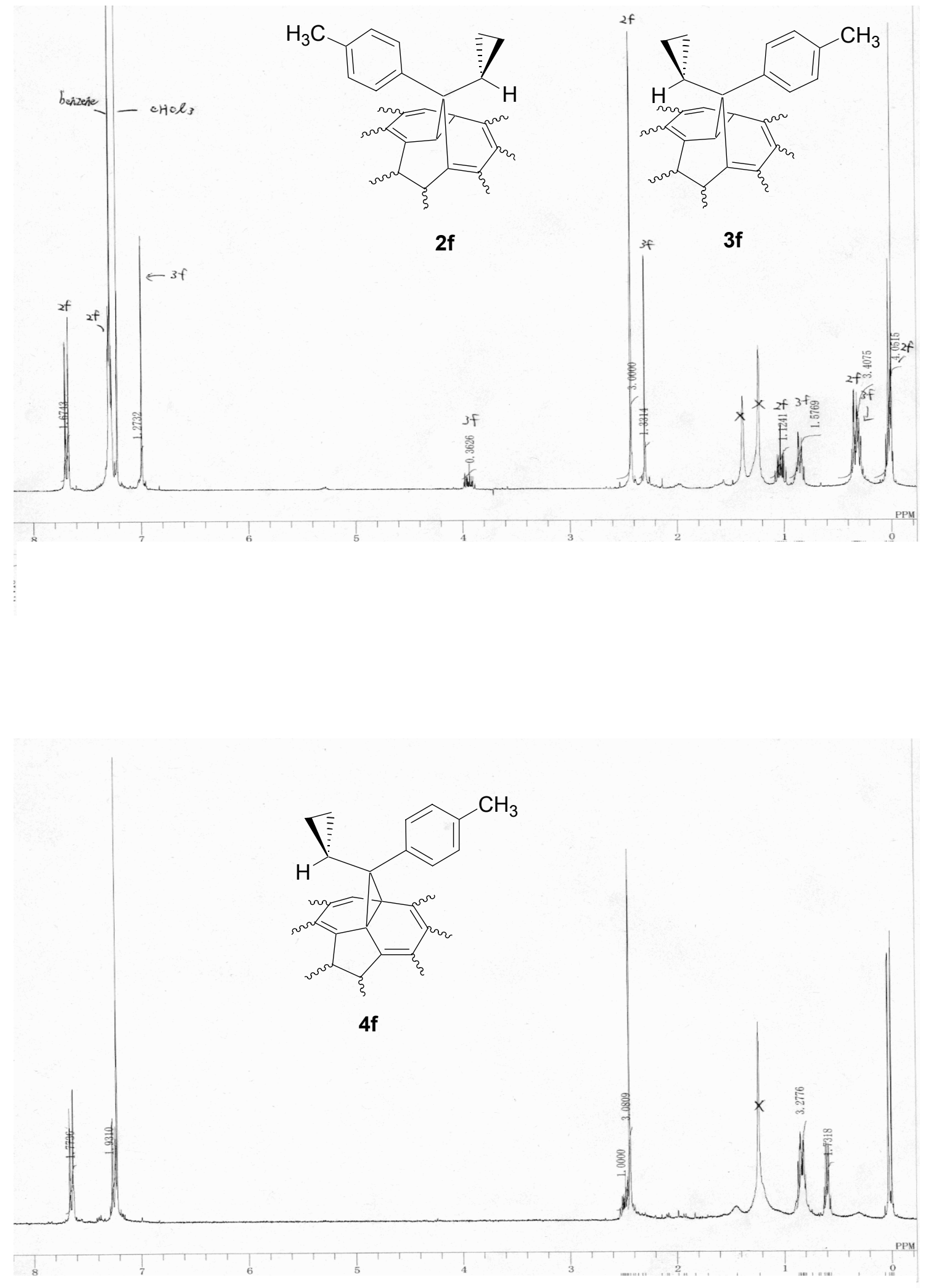

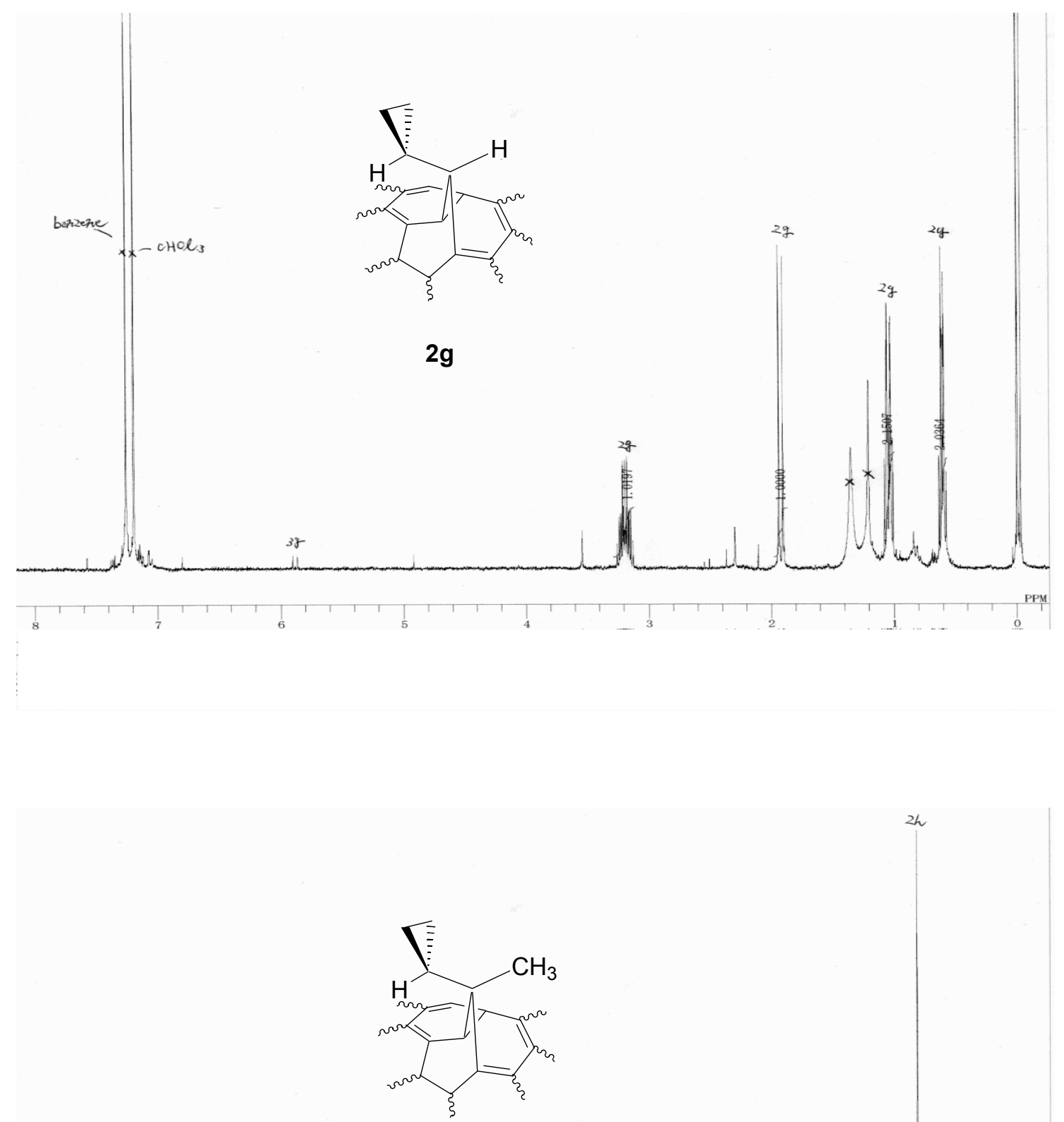

2h

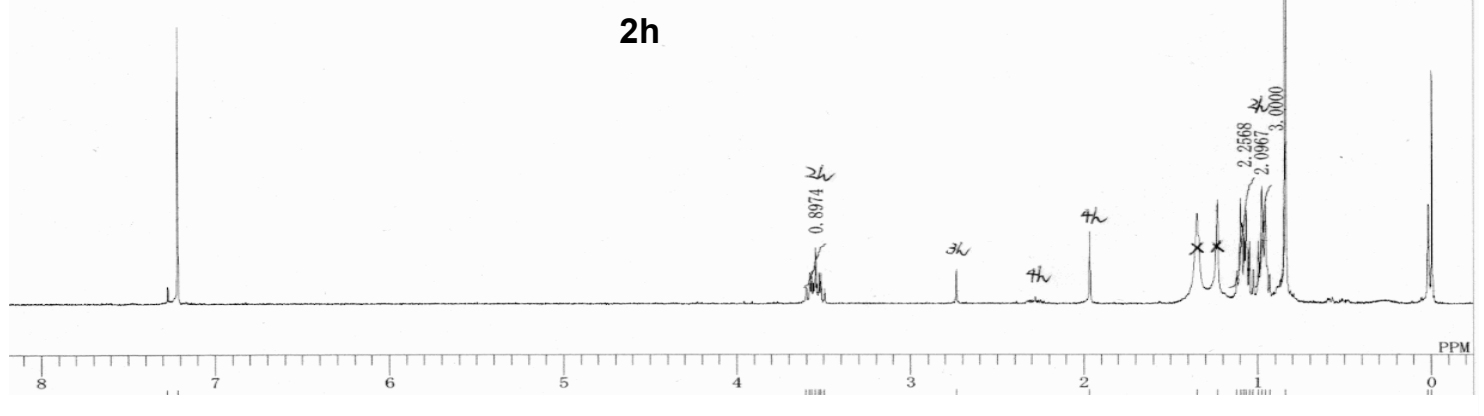



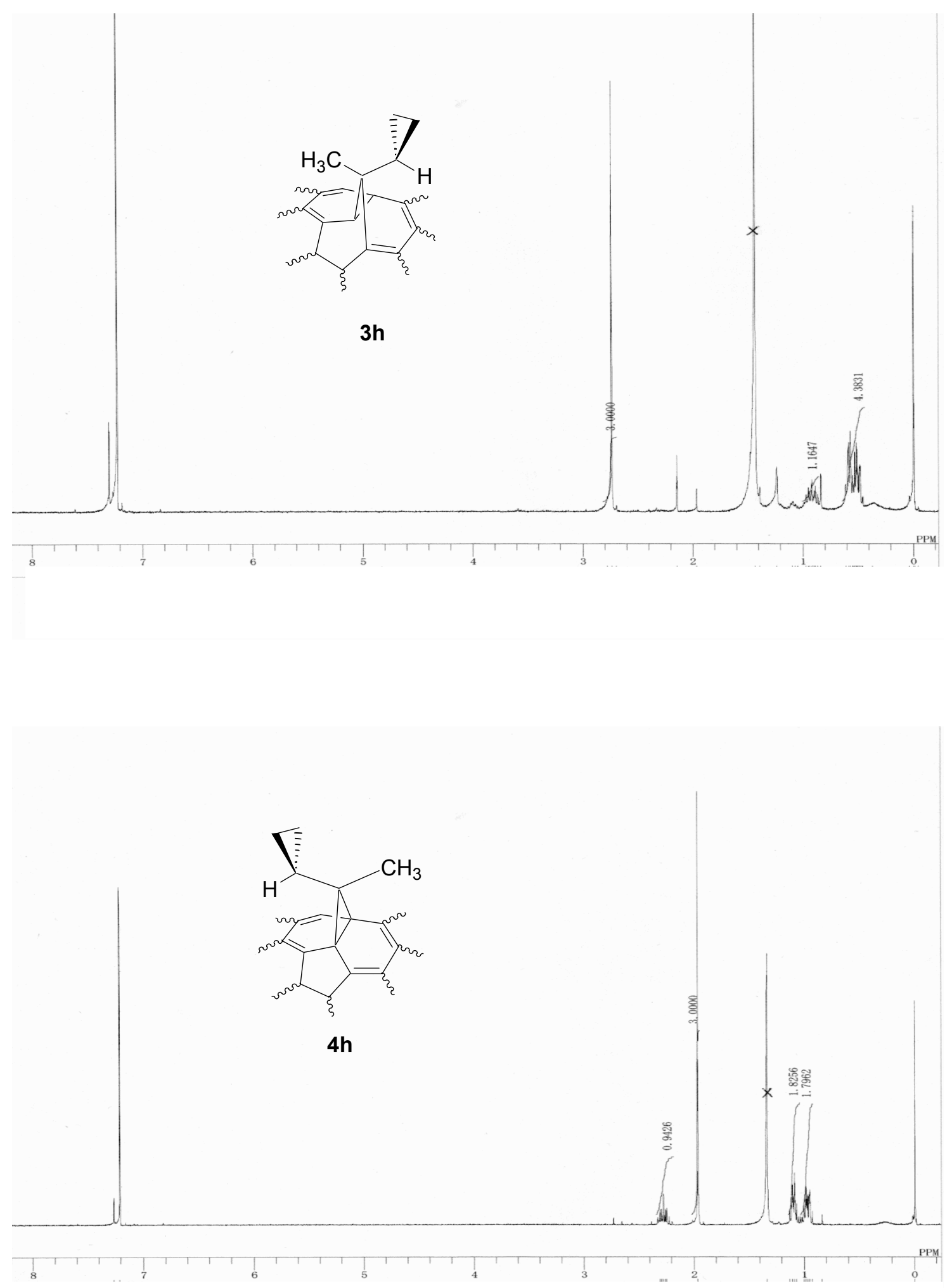
$2 \mathrm{i}: 3 \mathrm{i}=1.8: 3$
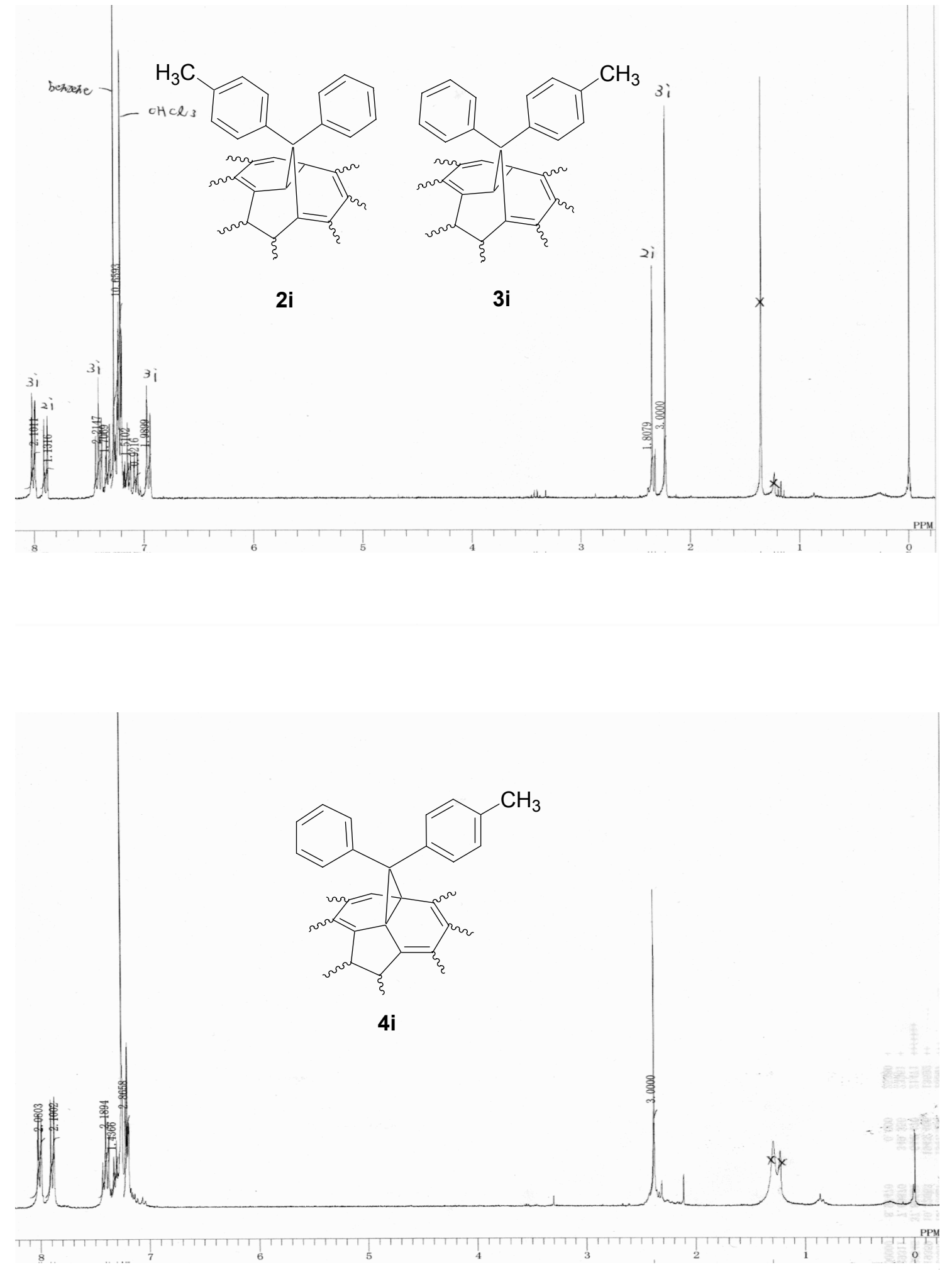
$2 \mathrm{j}: 3 \mathrm{j}=1: 4$
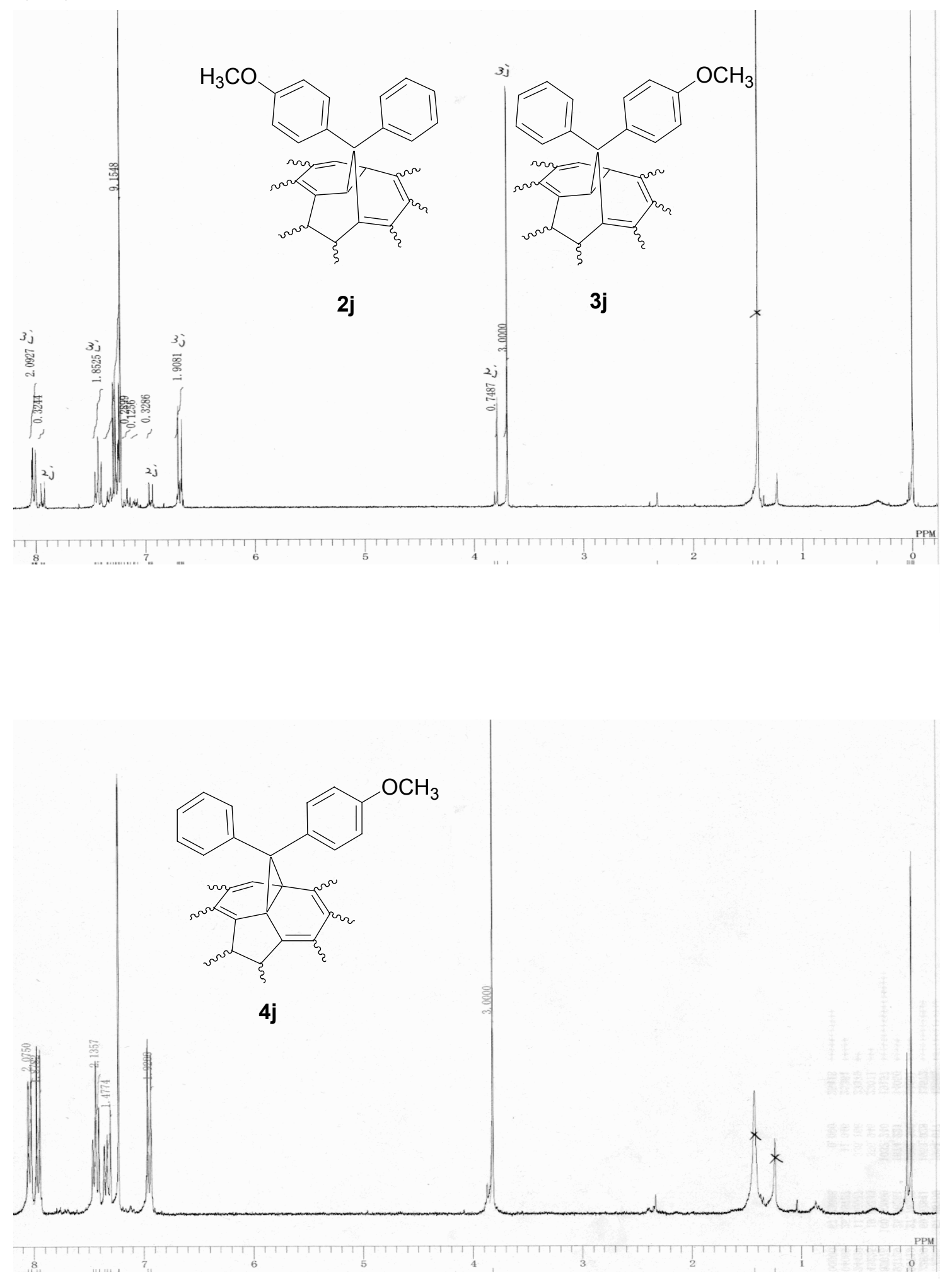

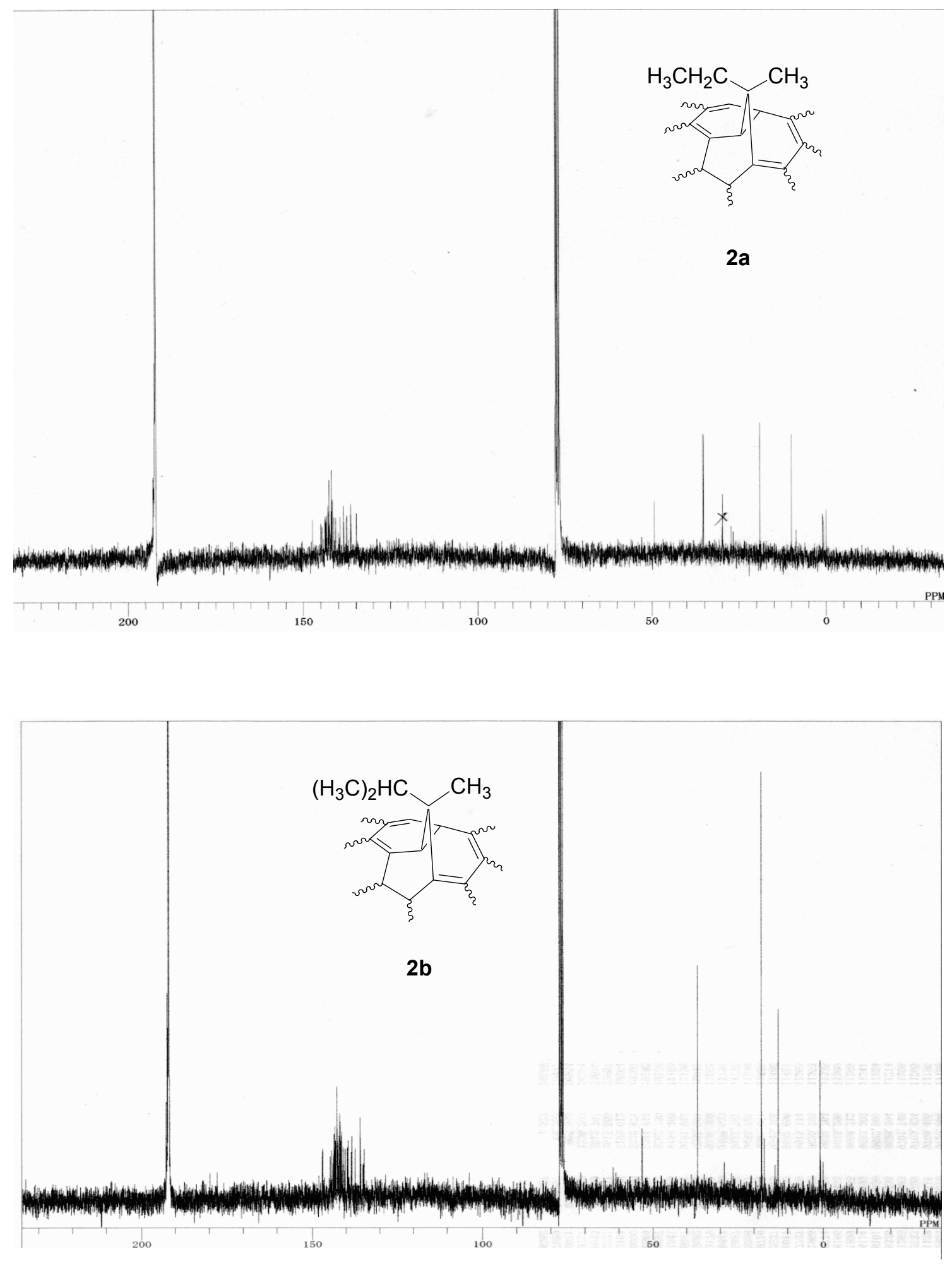


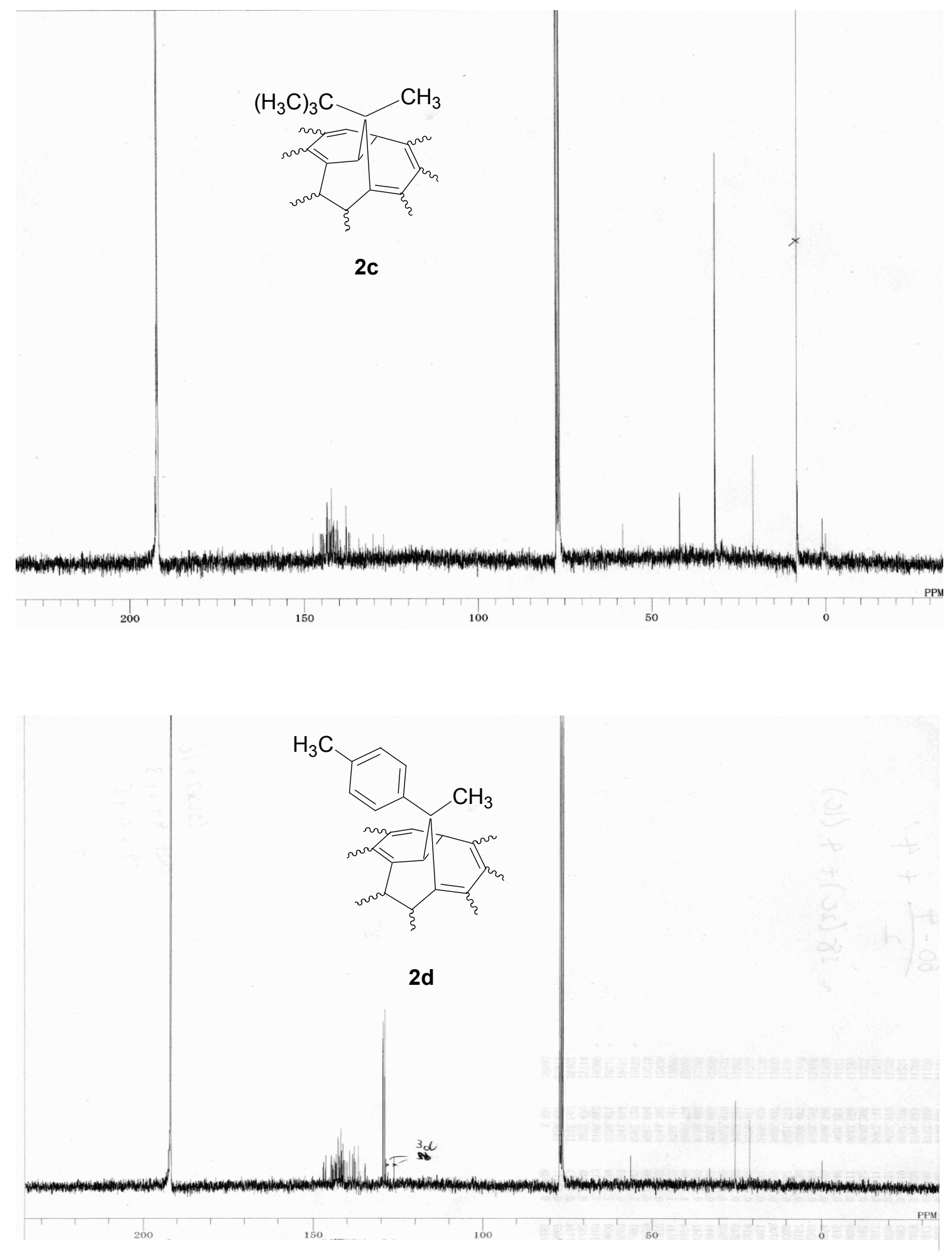



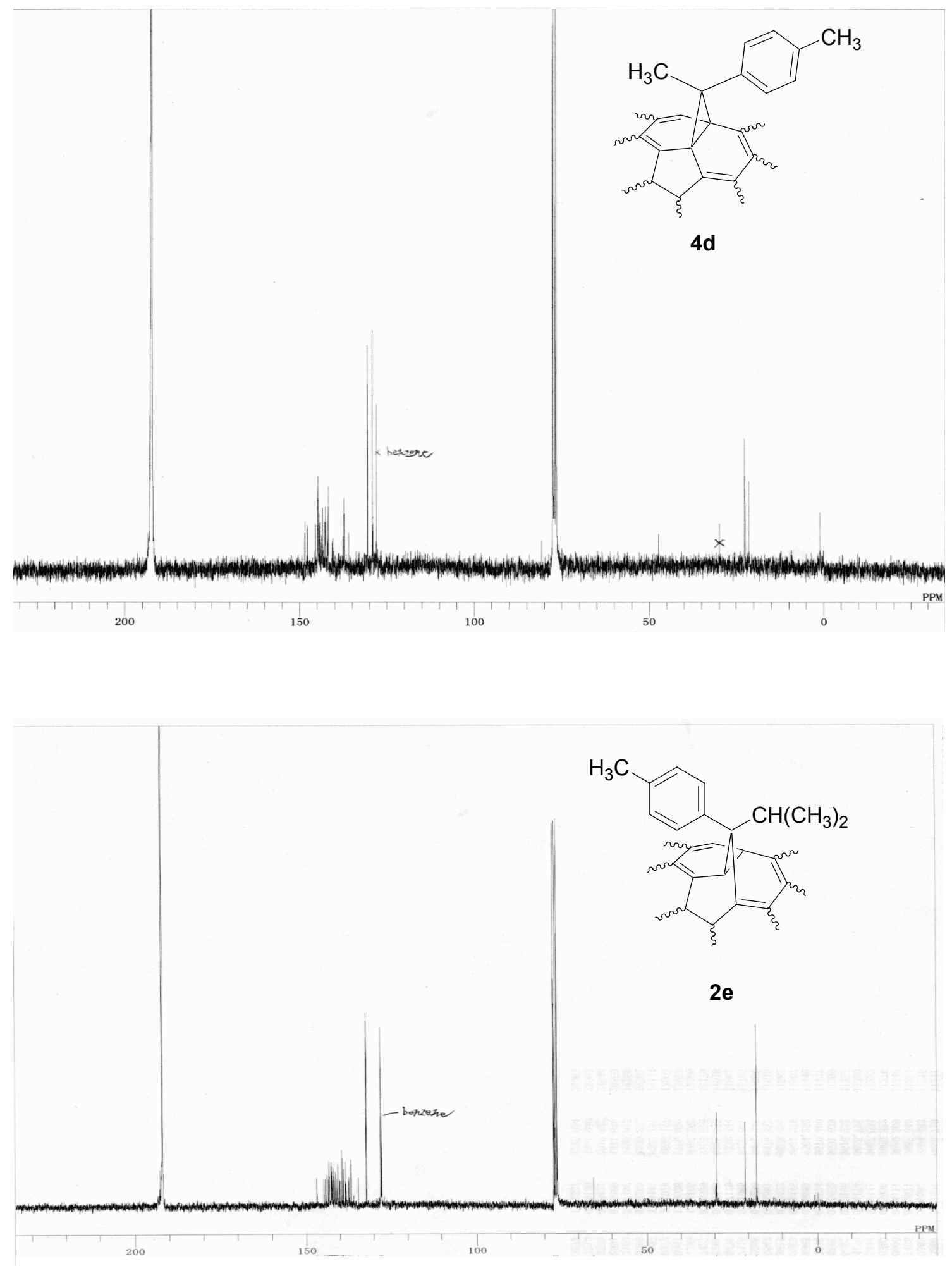

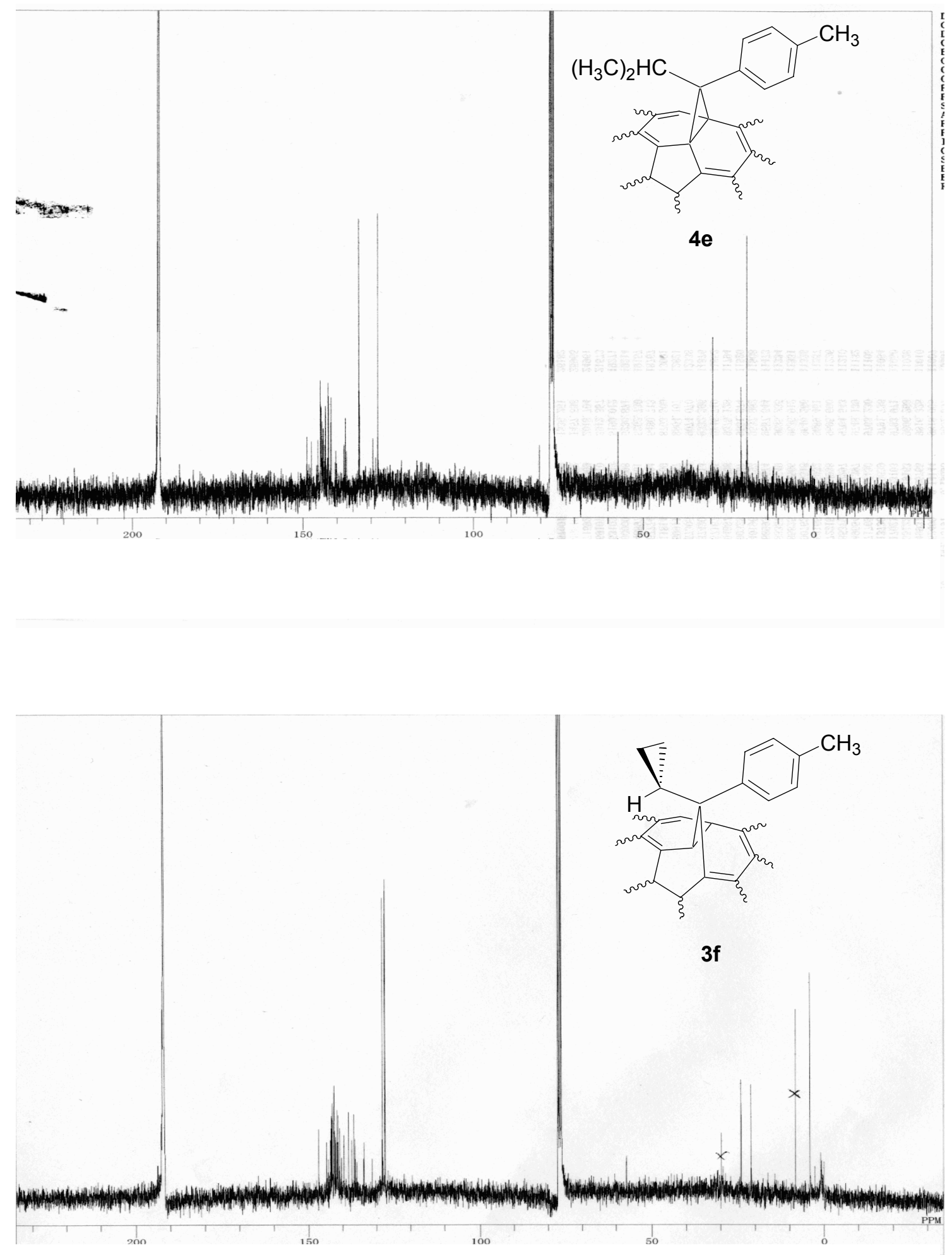

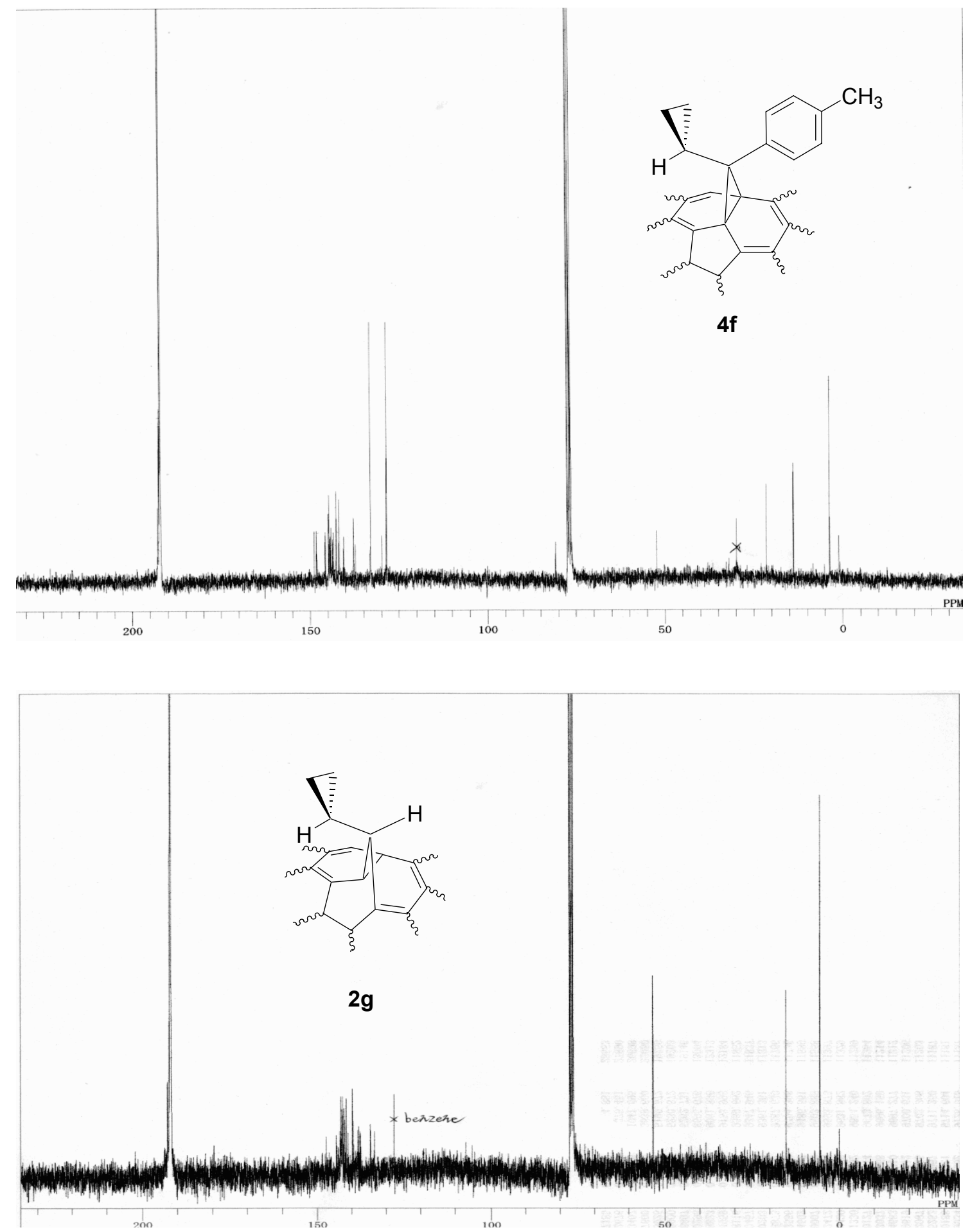

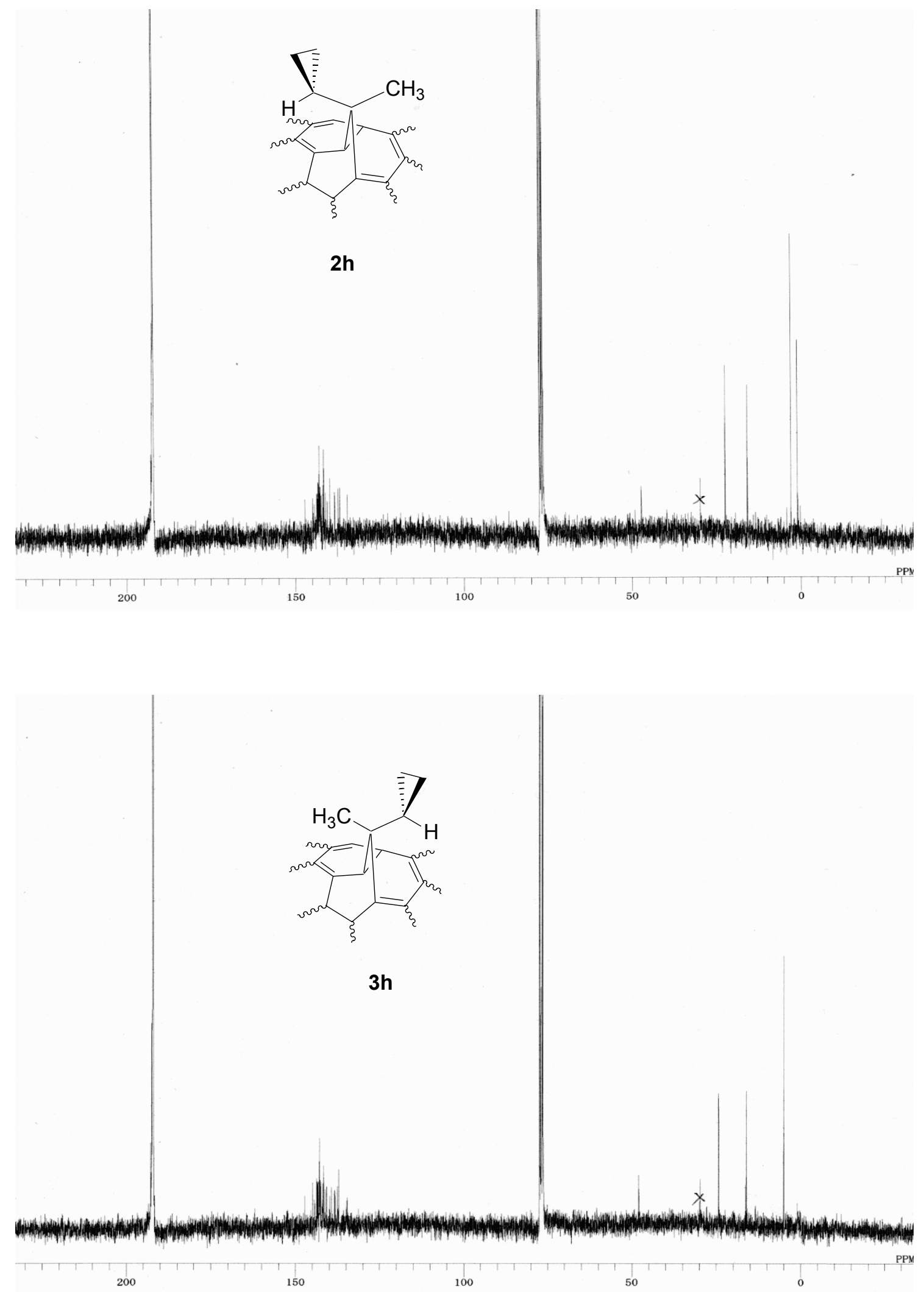

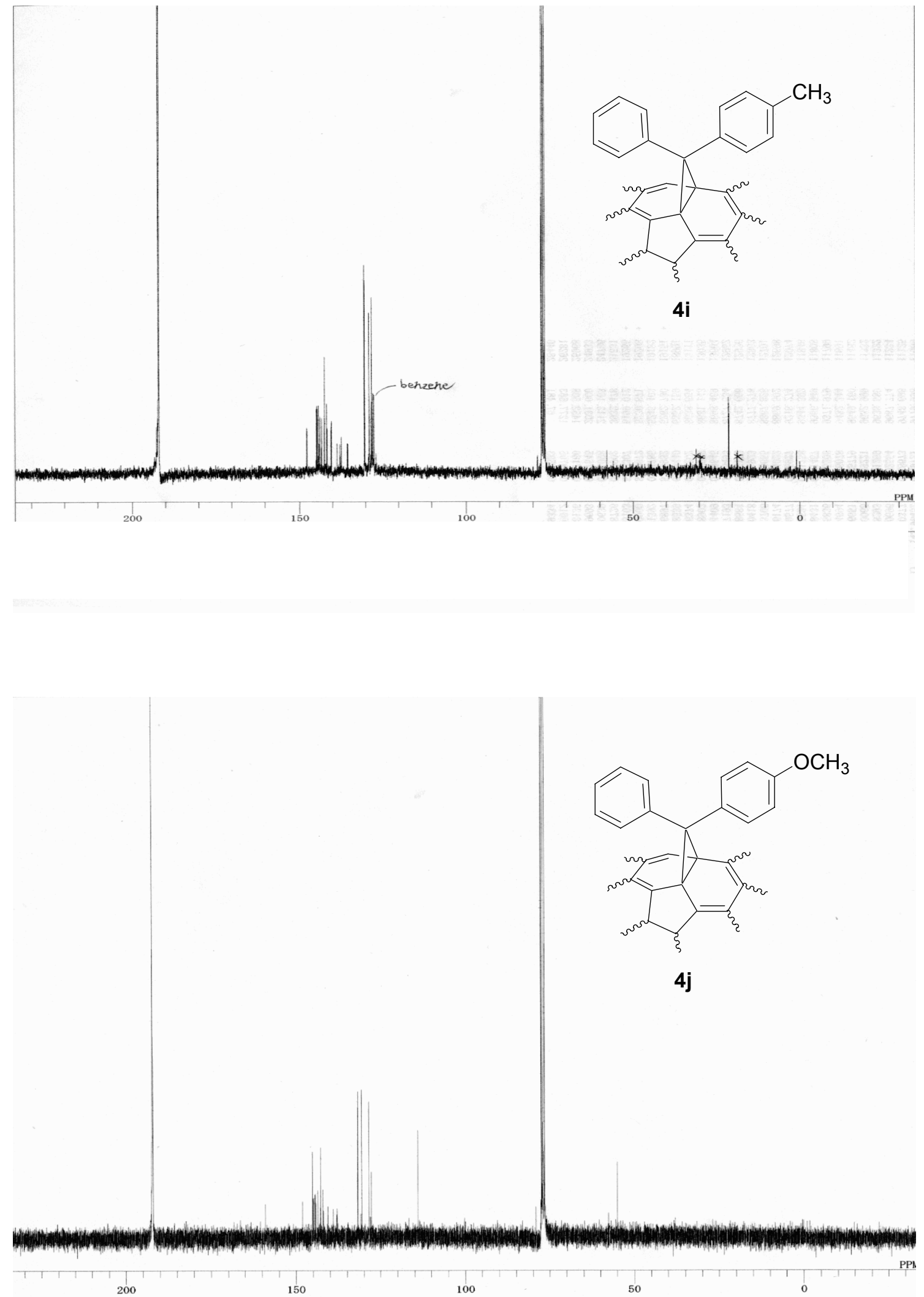
CH. 1 C.5 2.5 ATT 8 DFFS 9 90/0日/9日 07:17
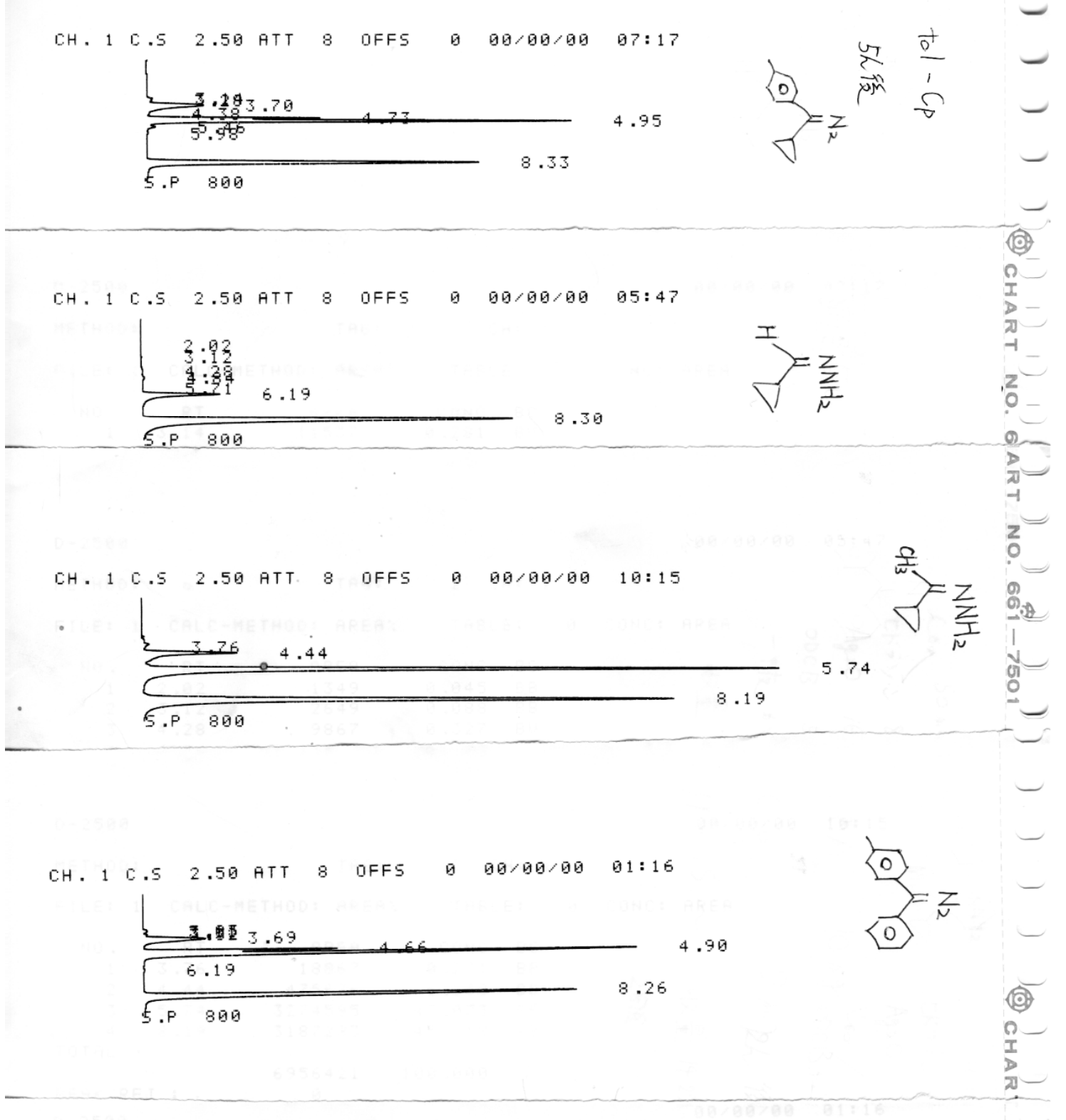

CH. 1 C.S 2.5 ATT 8 DFFS Q 90/0日/9日 $94: 41$

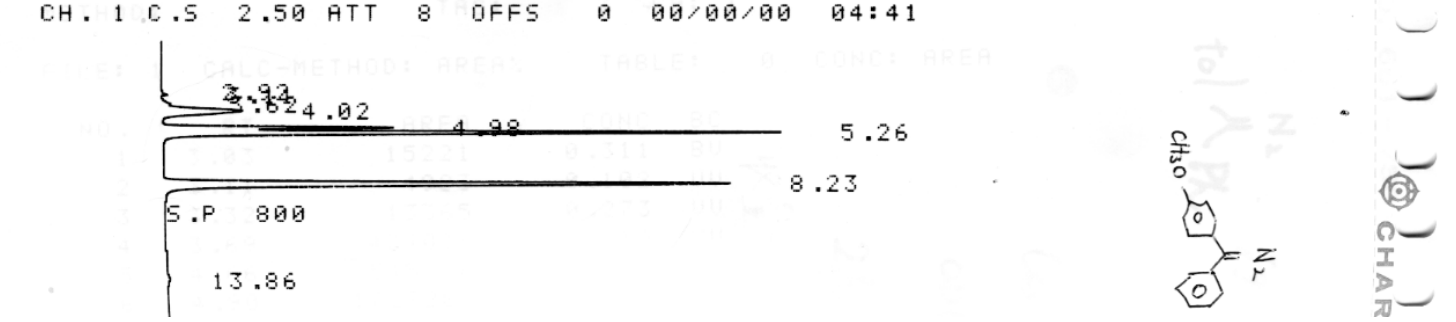


Product ratios

Entry 1

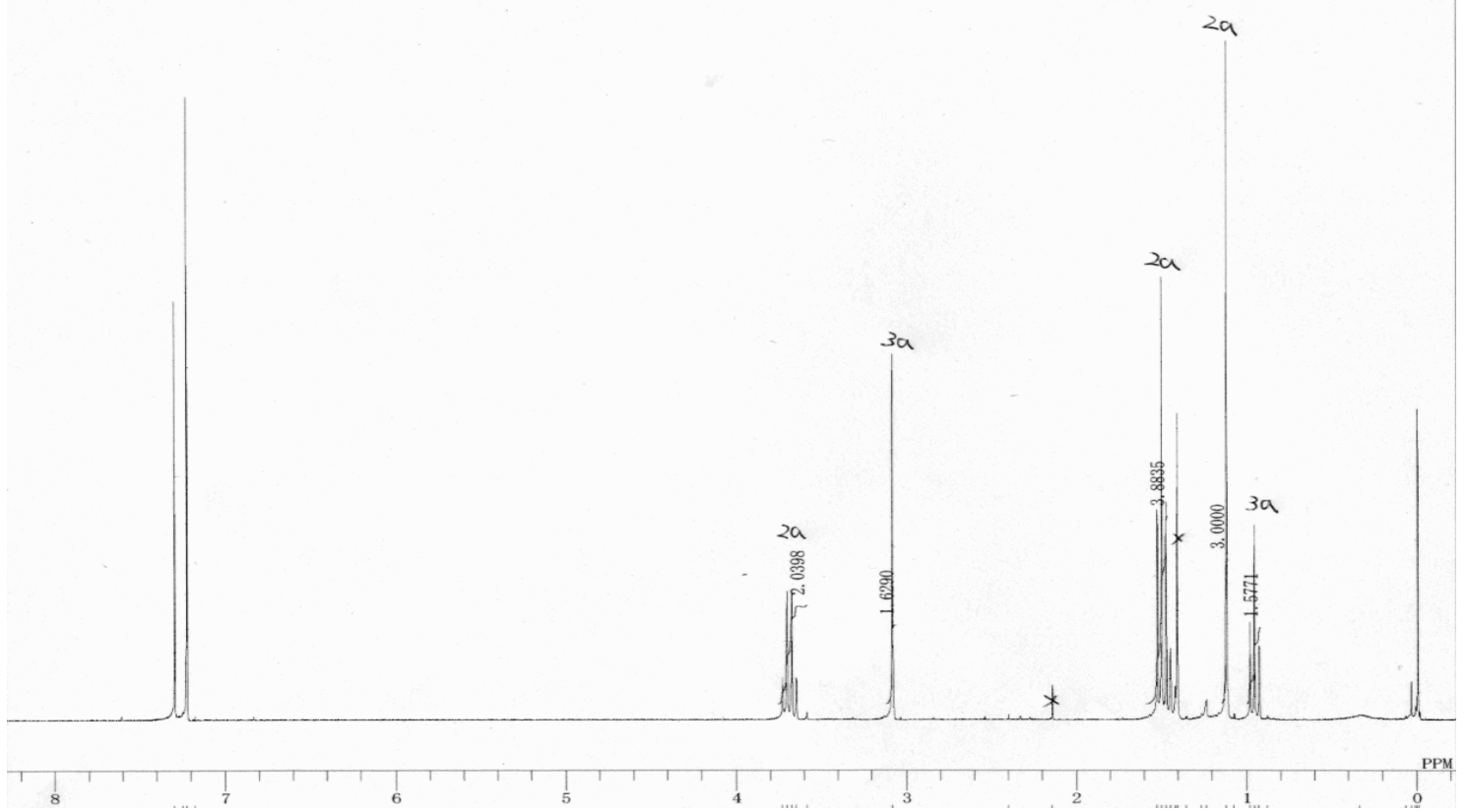

Entry 2

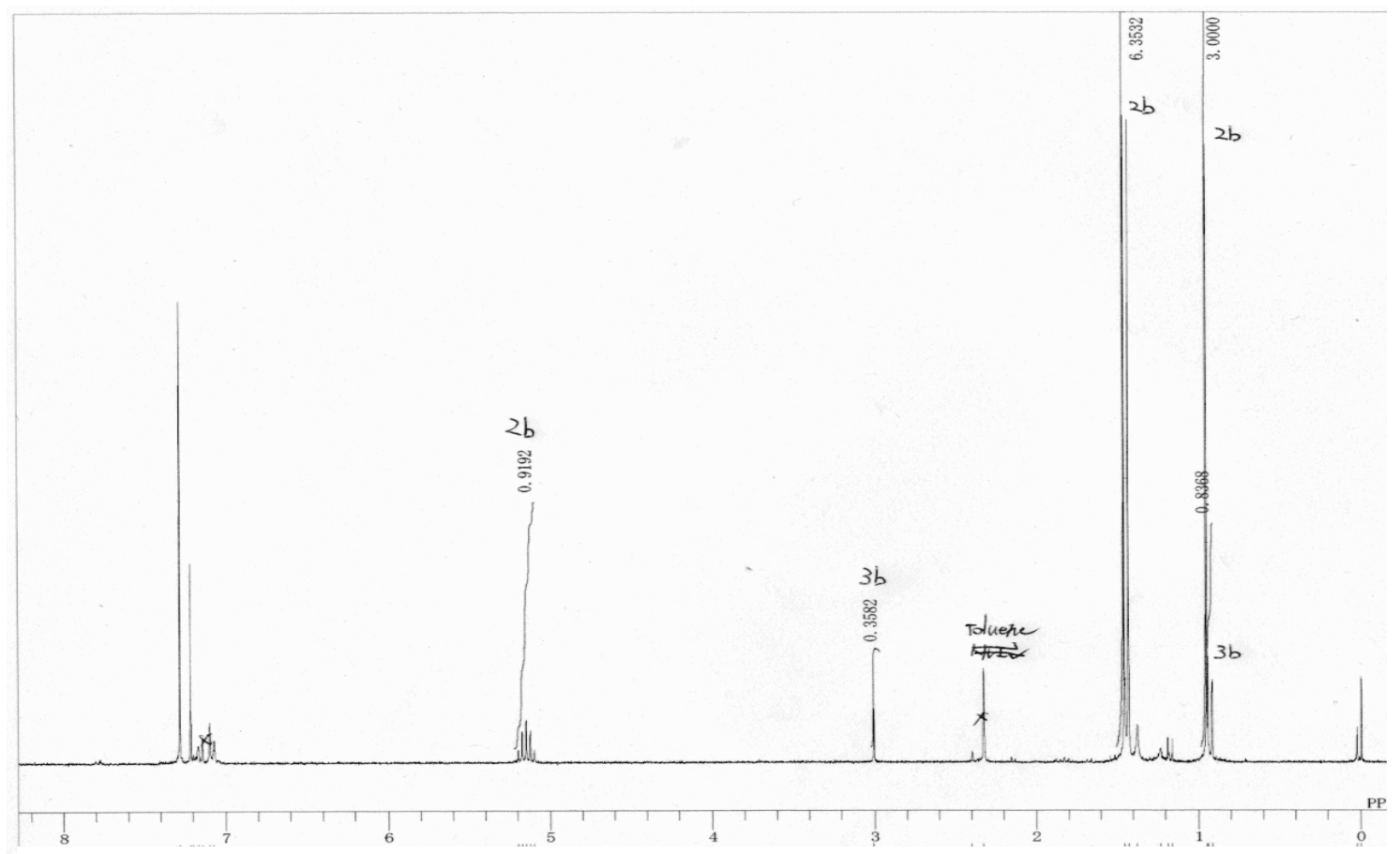


Entry 3

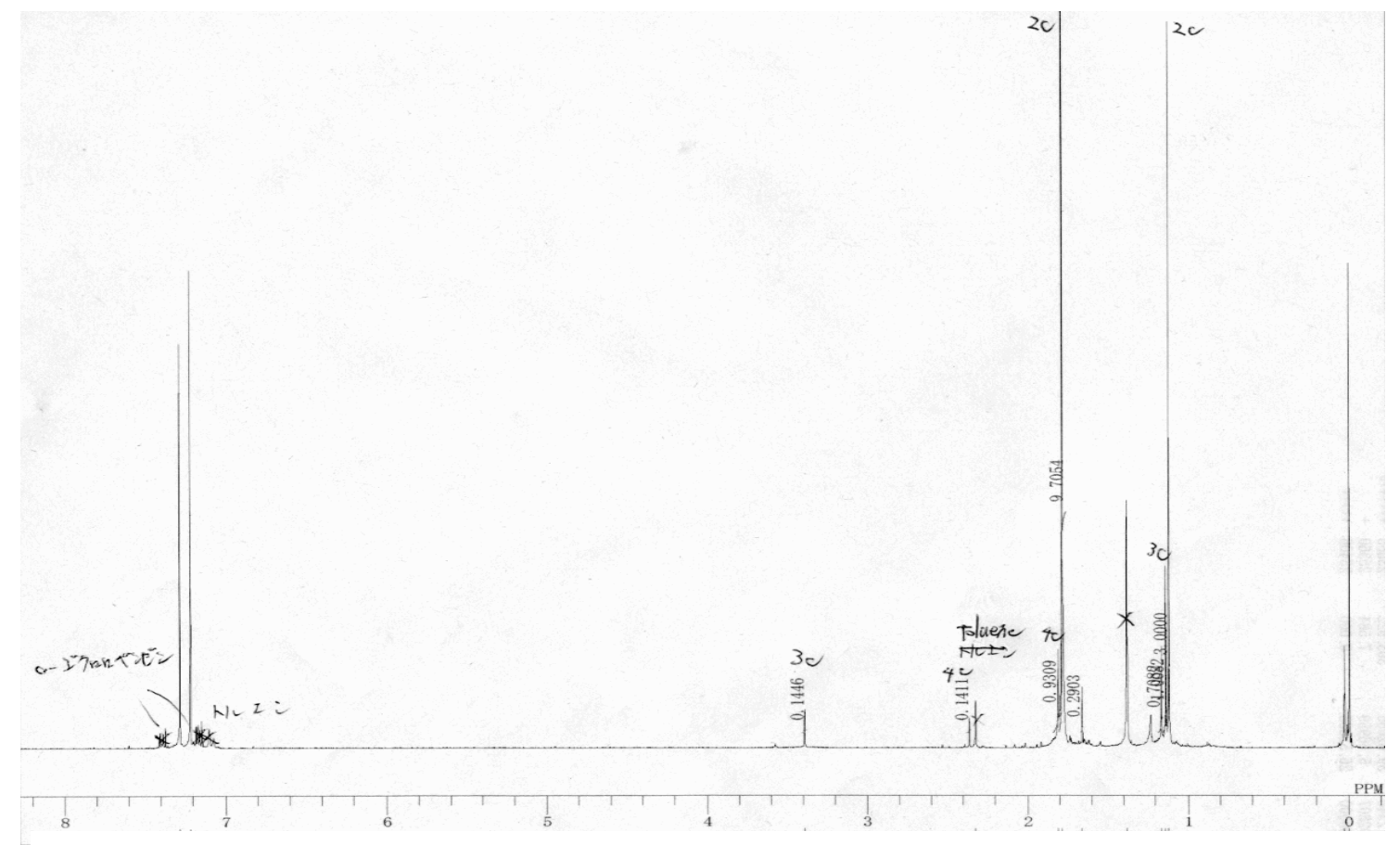

Entry 4

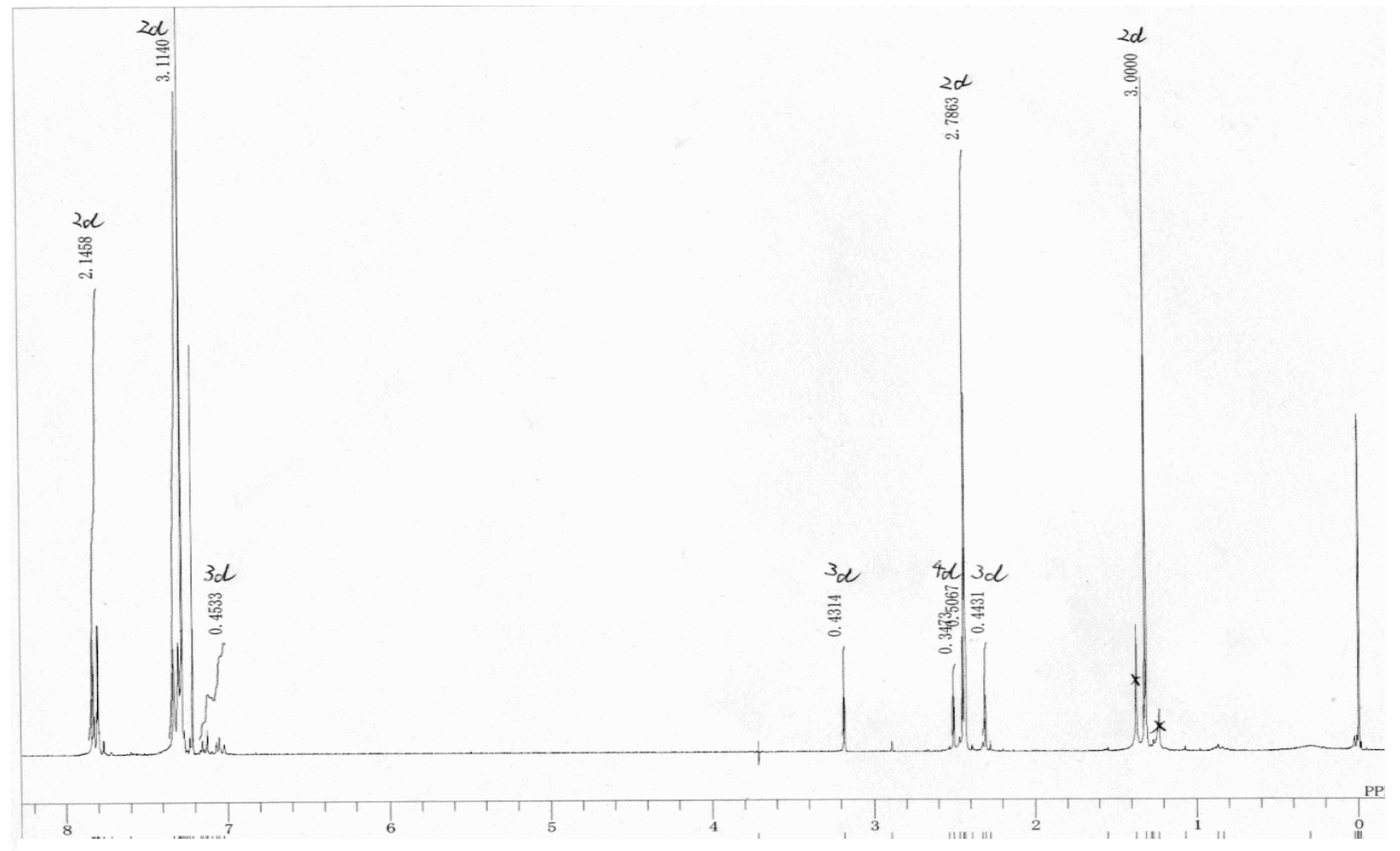


Entry 5

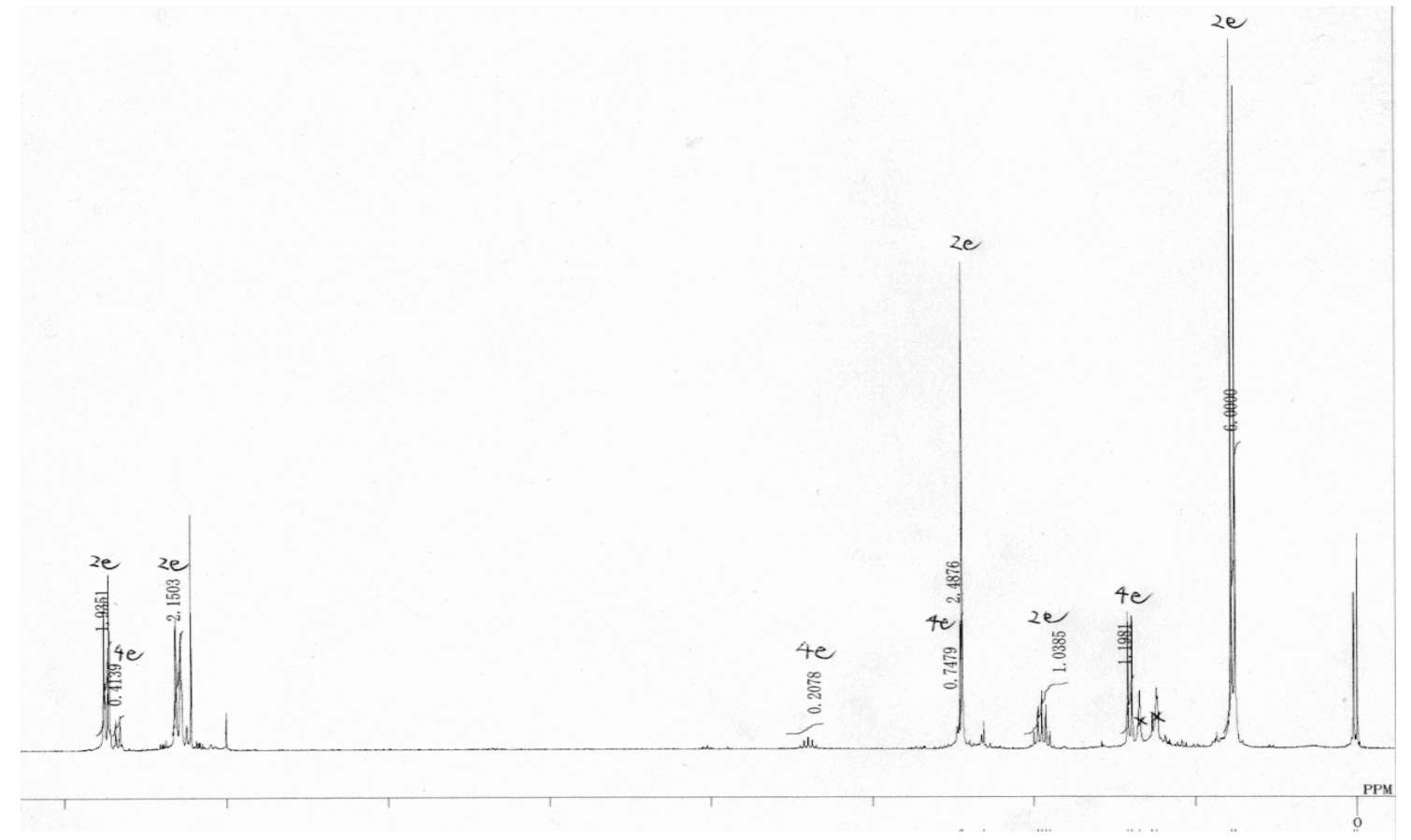

Entry 6

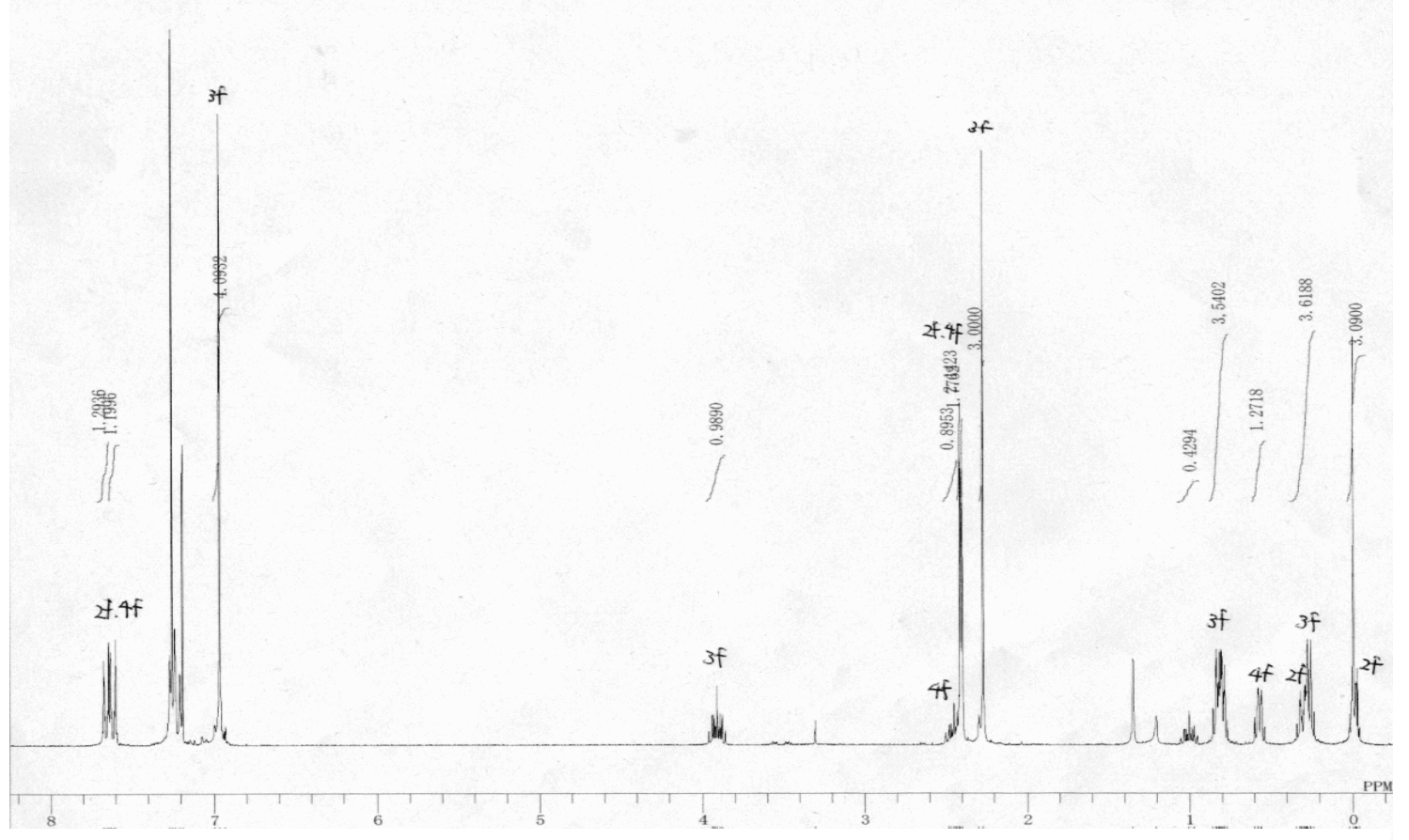




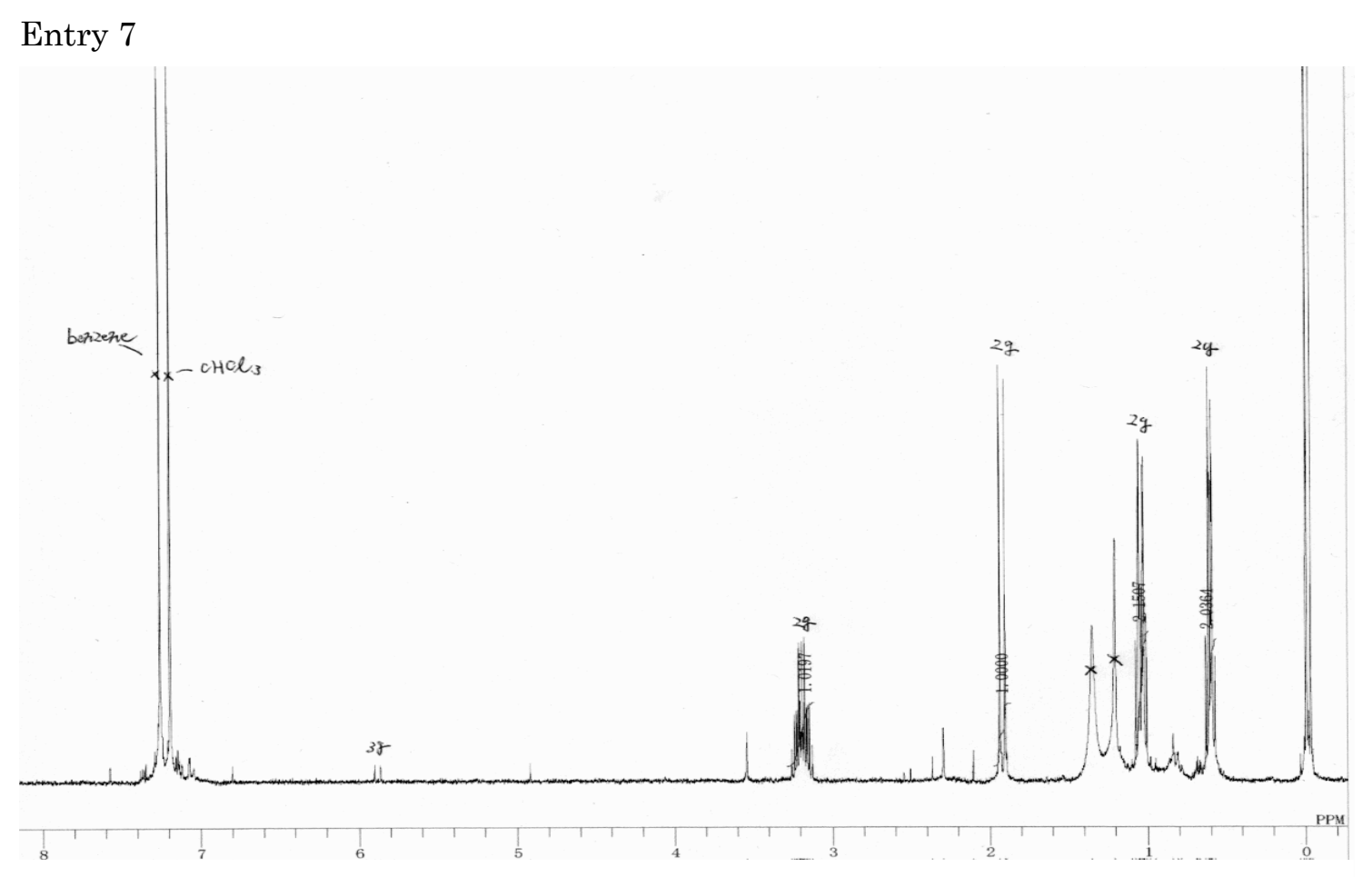

Entry 8

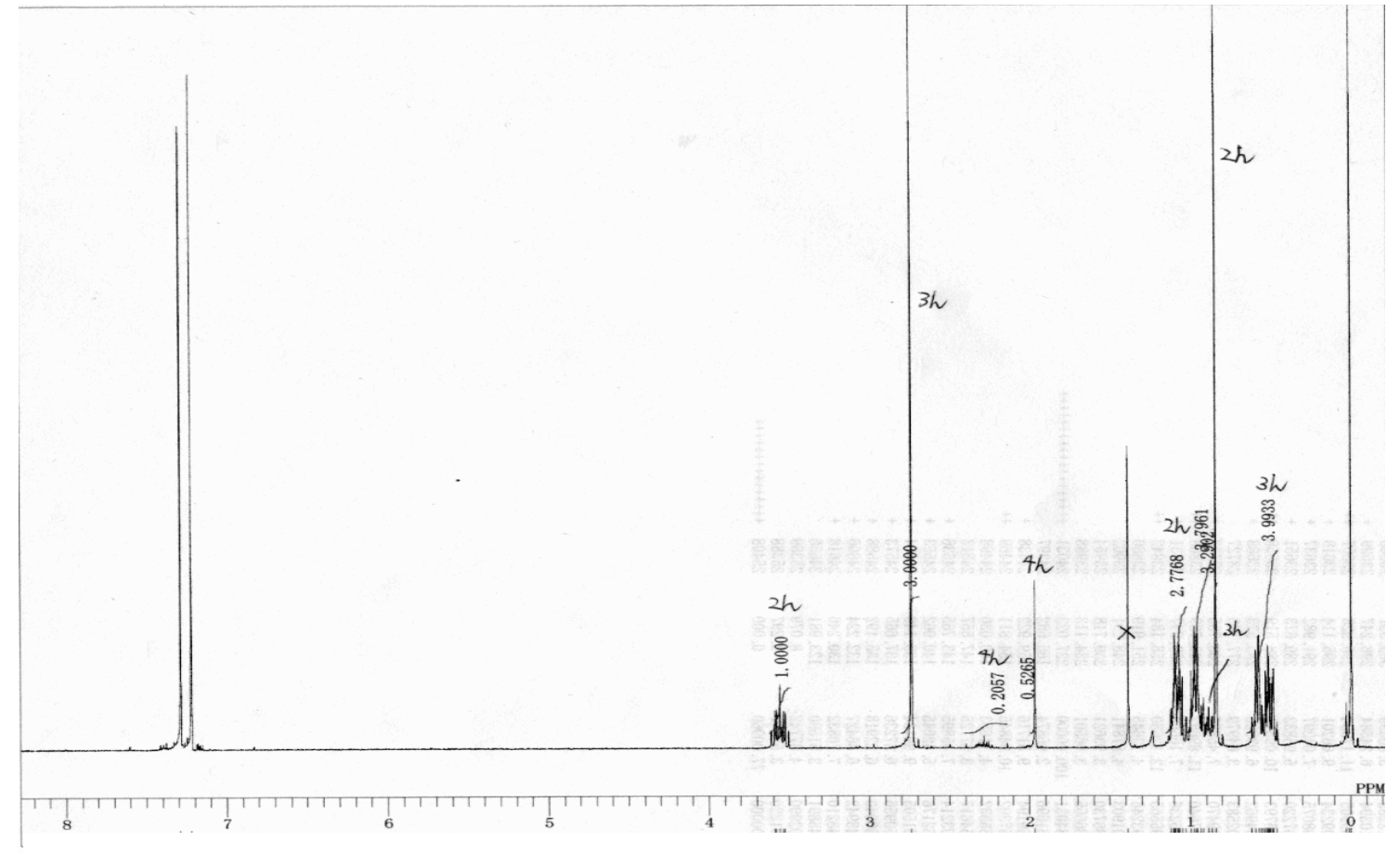




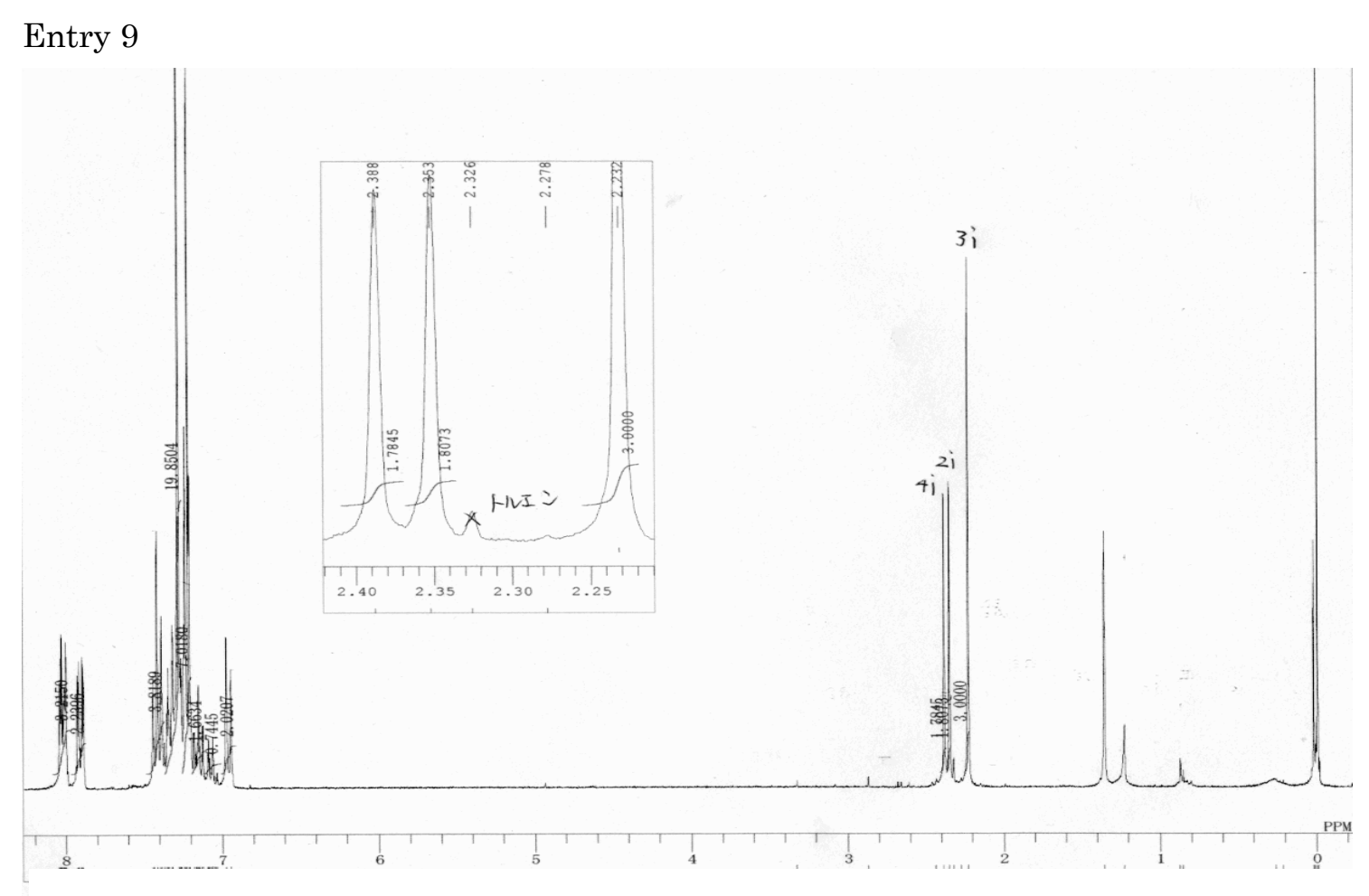

Entry 10

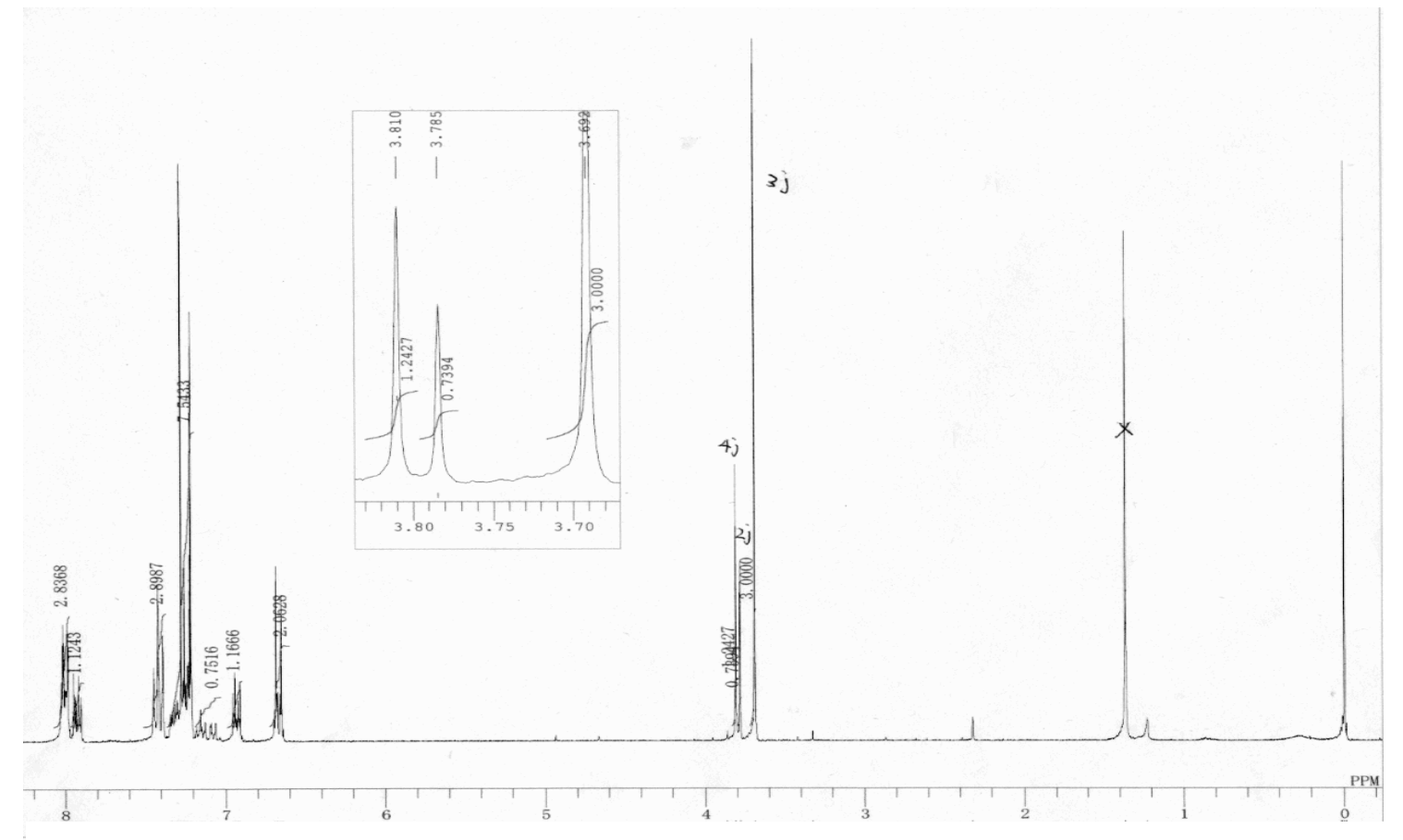


${ }^{1} \mathrm{H}^{-1} \mathrm{H}$ decoupling NMR spectra

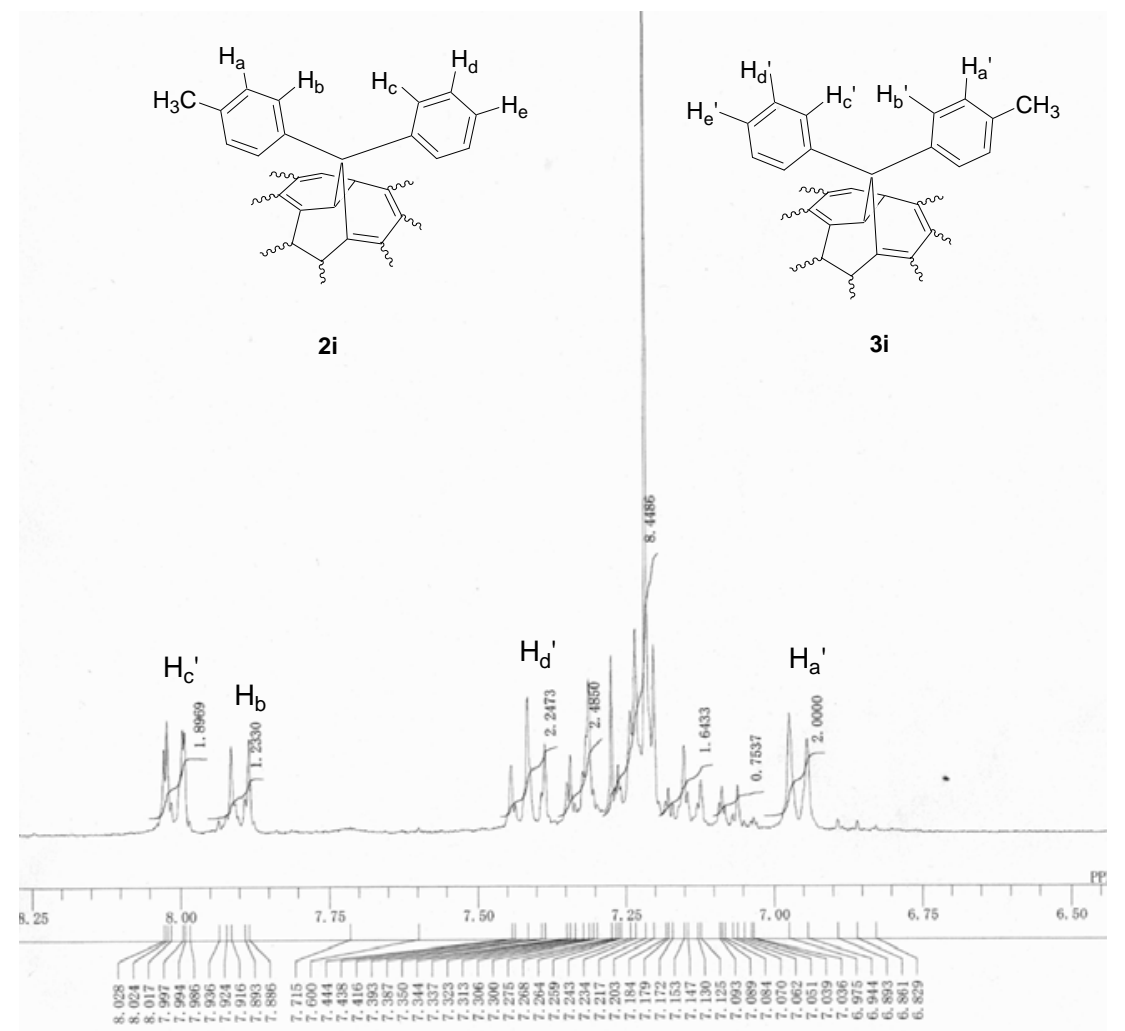

Ha' decoupling

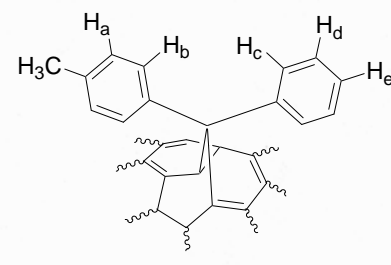

2i

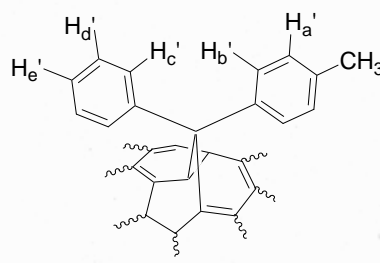

$3 i$

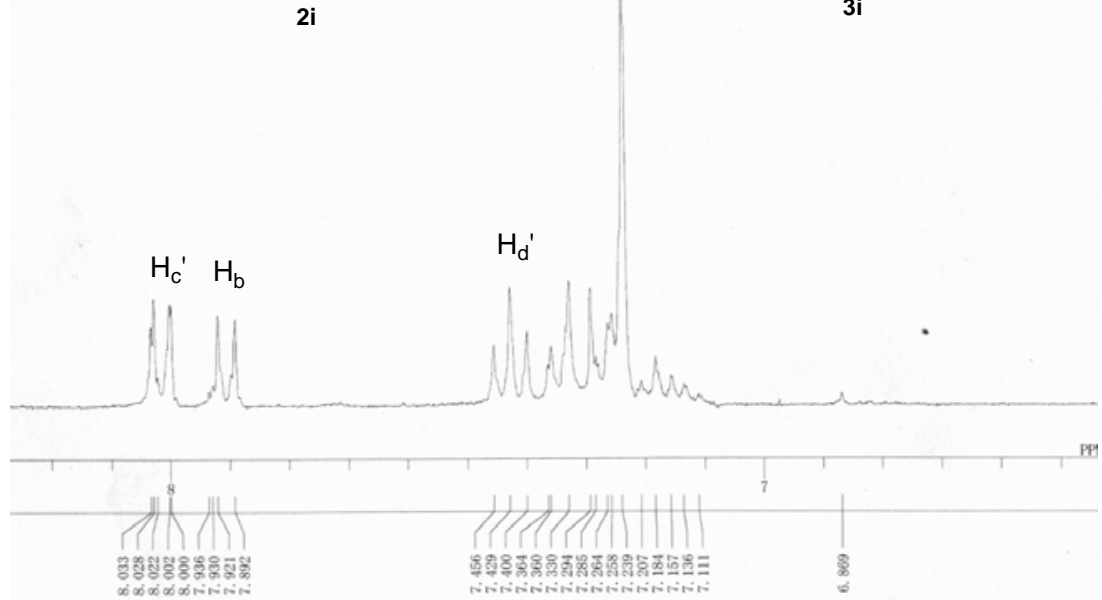


Hc' decoupling

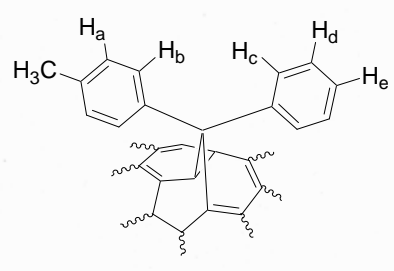

2i

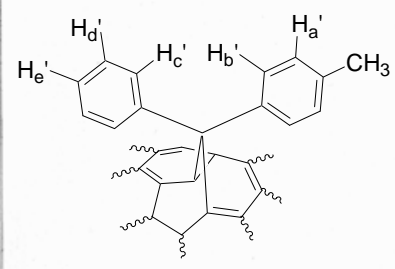

3i

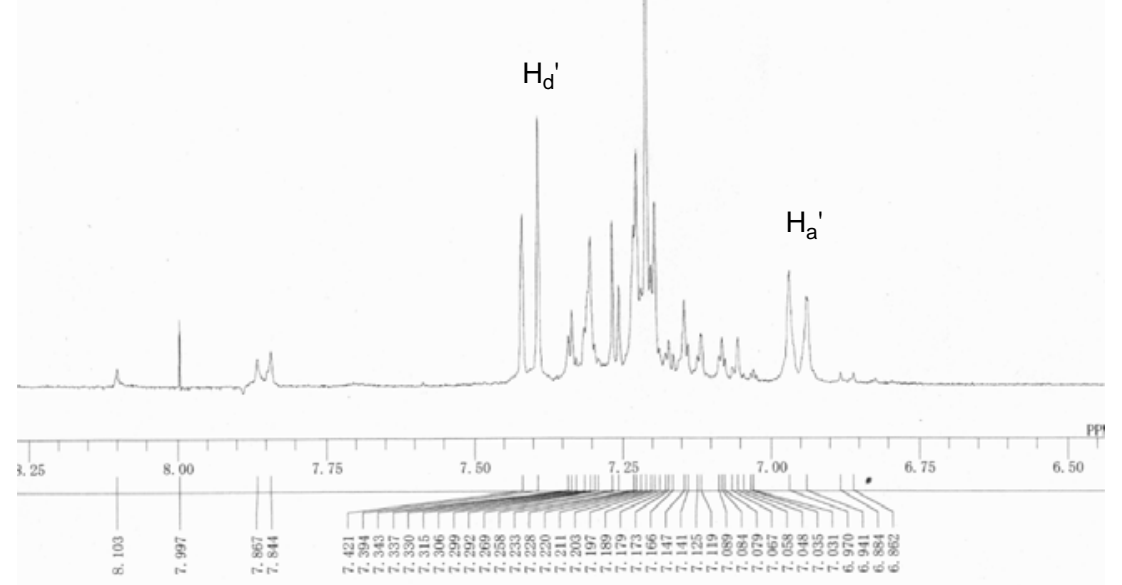


${ }^{1} \mathrm{H}^{-1} \mathrm{H}$ decoupling NMR spectra

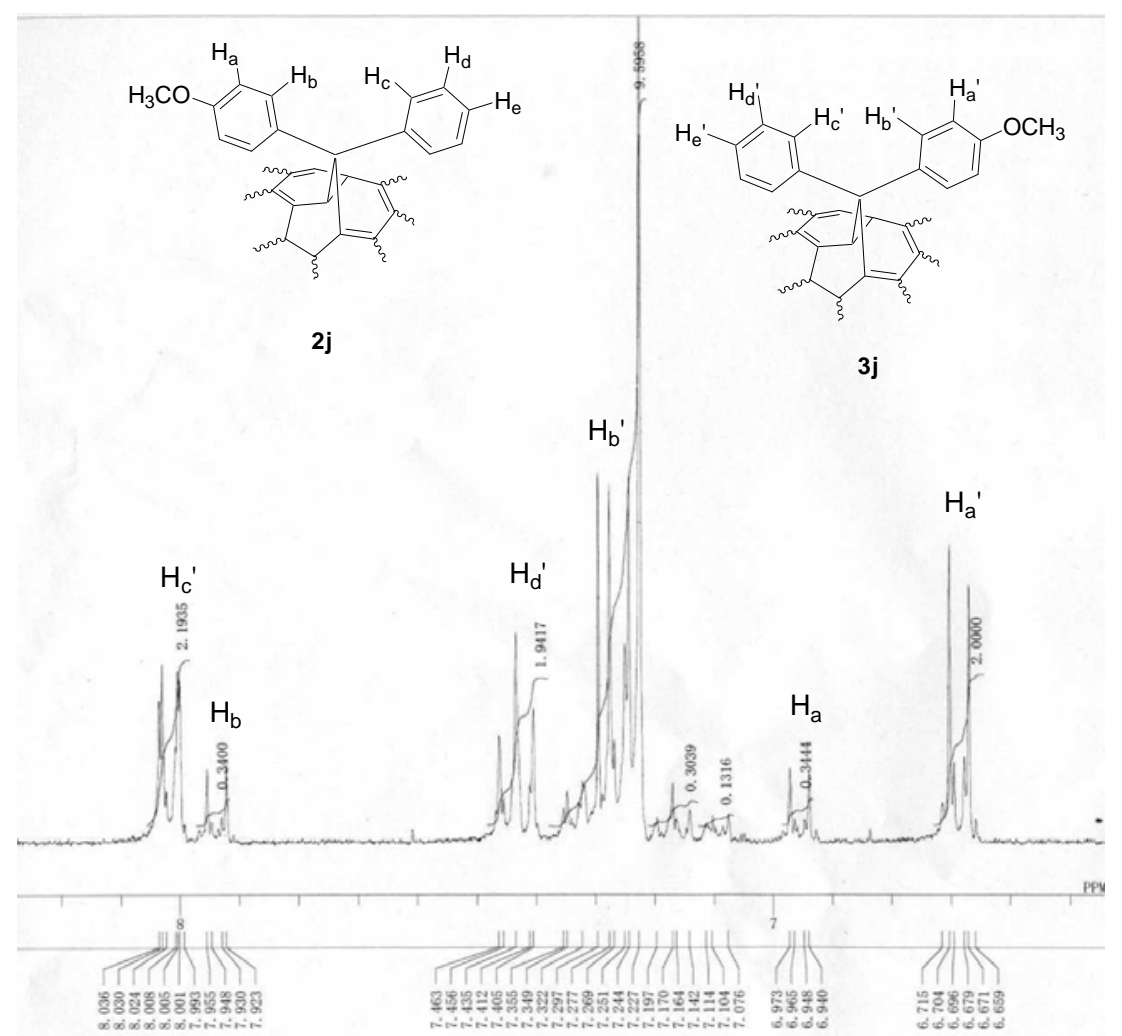

Ha' decoupling

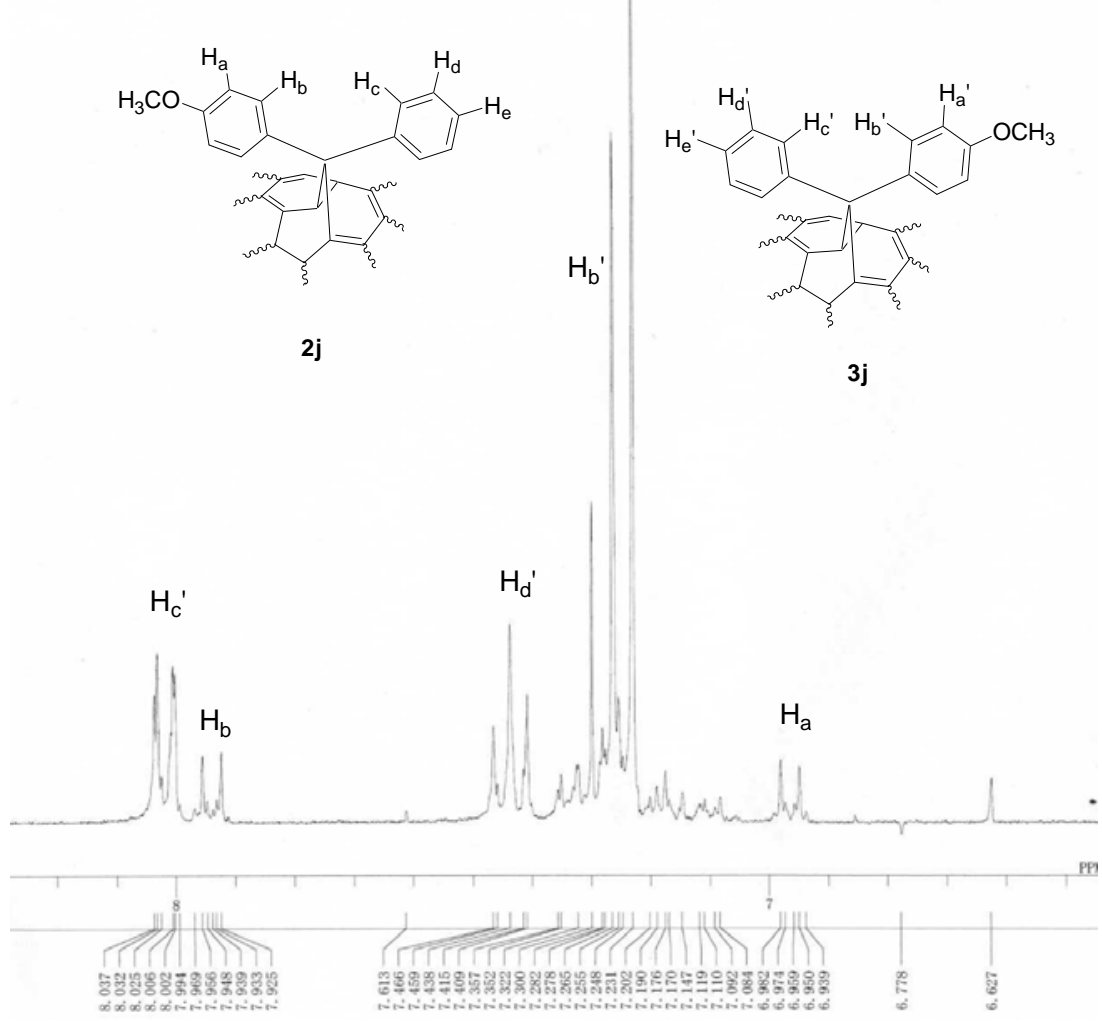


Ha decoupling

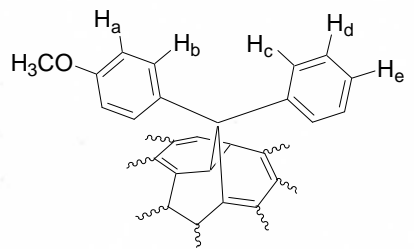

2j

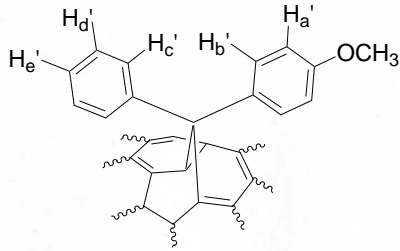

3j

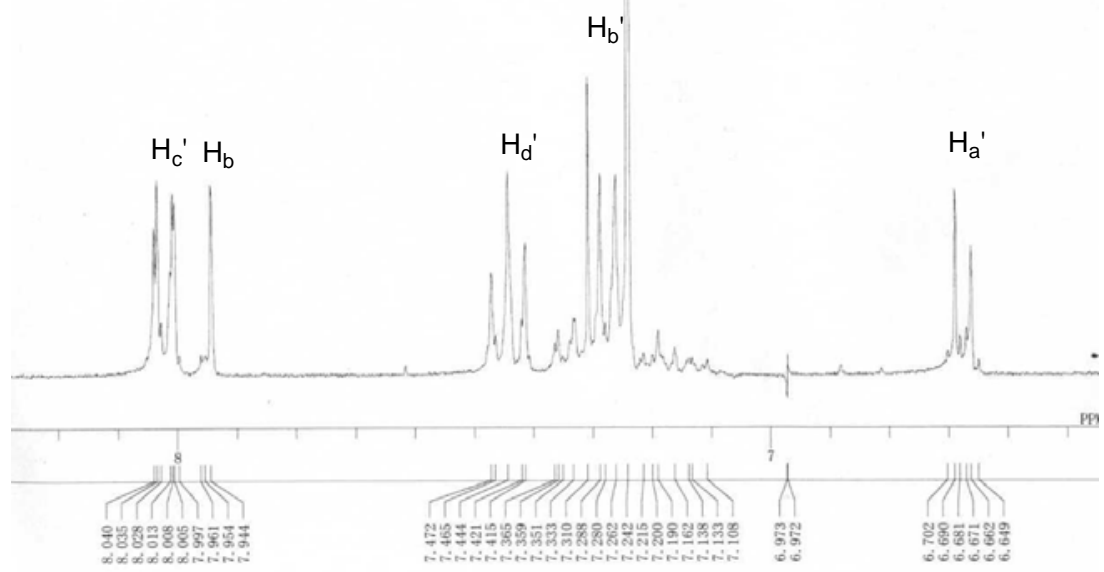

Hc' decoupling

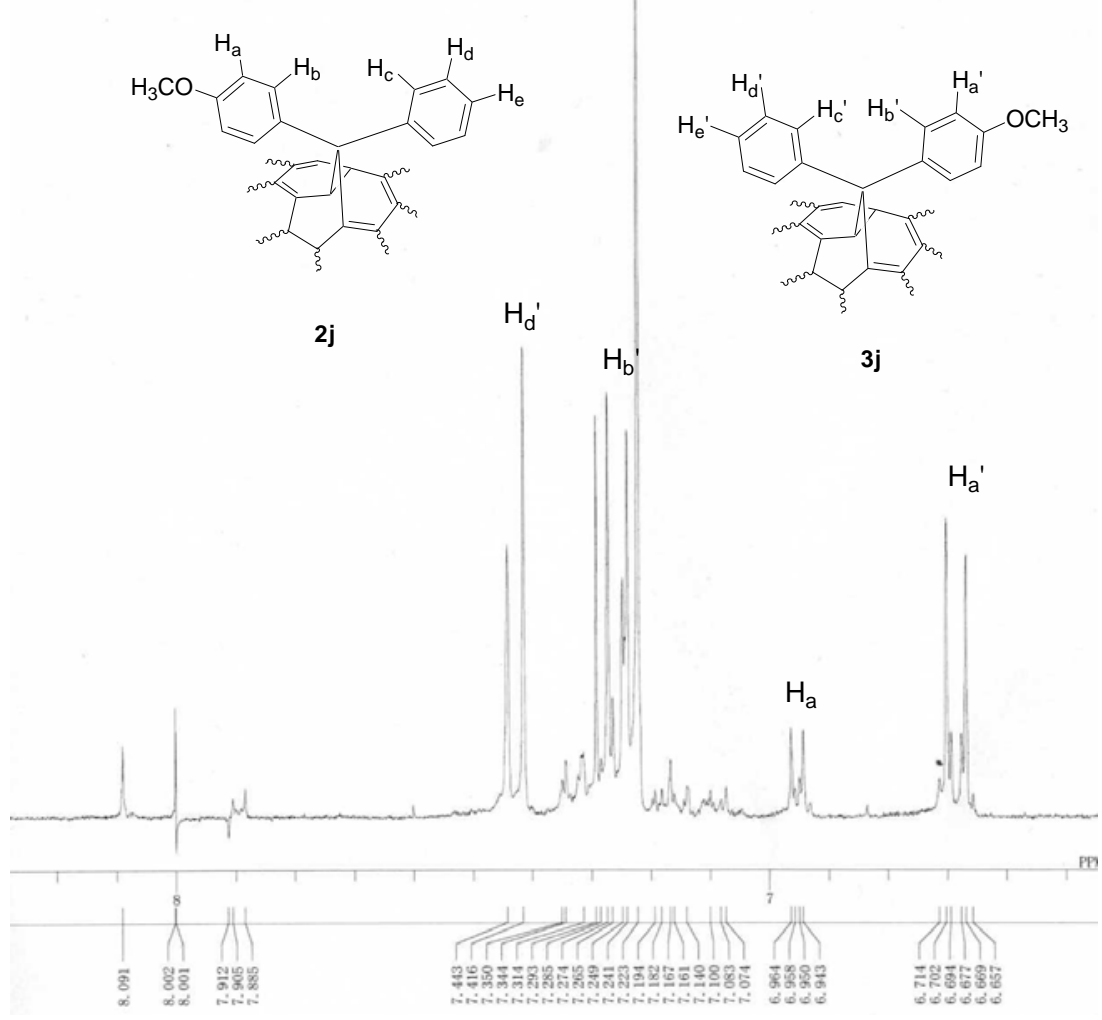

